\title{
A STUDY ON THE RELATIVE GROWTH IN THE BODY OF ALBINO RATS
}

\author{
$B y$ \\ KUNIO MOORI \\ Department of Anatomy, School of Medicine, Kyushu University \\ (Director, Prof. Mori) \\ Department of Histology, Kyushu Dental College \\ (Director, Prof. Saegusa)
}

Growth phases of experimental animals have been so far variously studied by many investigators. Donaldson (1924) was the first investigator who made detailed measurements on various bodily parts and organs in the growing albino rats. Later Huxley ('32) and Teissier ('34) studied particularly the problem of the relative growth in animals and they are credited to have devised an equation $\mathrm{y}=\mathrm{bx}^{\alpha}$ in which the whole or a part of the growing animal was represented by $\mathrm{x}$, another part or an organ by $\mathrm{y}$ and $\alpha$ represented the relative growth rate or the coefficient of relative growth. Recently reports on the relative growth have also been made in our country, of which Shimizu's was rather exhaustive. However, the report was based upon Shimizu's recalculation of the data coming from other investigators such as Negishi, Watanabe, Tappe and Donaldson, rather than upon the data obtained by himself from actual measurement of animals of his own breeding. The present report concerns with the study on the development of various parts of the body including the main internal organs which were collected from albino rats bred in the author's laboratory. The results of the measurement of these animals were used to calculate the relative growth rates of each bodily part and each organ against growth rate either of body length or of body weight.

\section{MATERIAL AND METHOD}

The embryos, sucklings and adults of the albino rat were used as the materials. The embryo to be studied for each stage of growth was collected in the following way : Male and female adult rats, one for each sex, were caged together for 12 hours, at the termination of which both were separated. The female was kept singly for another 12 hours, and embryos delivered from her meanwhile were designated as the first day embryos. Based on this dating embryos and sucklings were daily collected from 13th embryonic day on to the 20 th day after birth, and from then further on to the 50th day sucilings were collected once in 5 days. Besides these, adults animals were collected freely.

The measurement was workd on the following items at each growth stage of the animal. 
Embryo :

1) Head to tail length

2) Body weight

Suckling and Adult :

3) Head to tail length

4) Body length

5) Body weight

6) Length between lower lip and anus

7) External orbital breadth

8) Internal orbital breadth

9) Breadth of rima palpebrae

10) Mouth breadth

11) Maximum face breadth

12) Maximum head length

13) Maximum head breadth

14) Length of mandibular angle
15) Height of ramus mandibularis

16) Length of upper extremities

a) Humerus

b) UIna

c) Radius

17) Length of lower extremities
a) Femur
b) Tibia

18) Length of intestine

19) Heart weight

20) Lung weight

21) Stomach weight

22). Liver weight

23) Kidneys weight

24) Spleen weight

25) Thymus weight

Method

Calipers were used for the measurement of length, and beam-balance or, if necessary, an analytical balance was used for the measurement of weight. In the study of relative growth the equation $\mathrm{y}=\mathrm{bx}^{\alpha}$ was utilized following the previous investigators. In the postnatal animals the growth rates in body weight, in lengths of body and four extremities, in lengths of various parts of head and in lengths of extremity bones were studied against the body length, and the weights of various internal organs were studied of the growth rates against the body weight. In the embryo of the animal the growth rate of body weight against the head to tail length was studied. The data presented here express the mean values for the respective part or item in the respective growth stage of the animal. Here $\mathrm{x}$ represents body length or body weight, and y represents each part of the body or each internal organ. The relative growth rate $\alpha$ and $\log b$ were calculated by the method of least squares. The value thus obtained were further studied on the logarithmic graphs.

\section{RESULTS}

The results obtained for each item of measurement were shown by Tables and Figures together with brief accounts of the developmental phases involved.

1) Head to tail length of the embryo.

The length increases slowly from the 13 th to the 19 th embryonic day when it reaches $1.63 \mathrm{~cm}$. By the 20 th embryonic day, however, an abrupt increase takes place attaining as much as $2.49 \mathrm{~cm}$, an increase shared also by body weight growth simultaneously. (Table 1, Fig 1)

Table 1

\begin{tabular}{r|r|r|r|r|r|r|r}
\hline Days & \multicolumn{1}{c|}{13} & 14 & 15 & 16 & 17 & 18 & 19 \\
\hline $\mathrm{M} \pm \mathrm{m}(\mathrm{cm})$ & $0.86 \pm 0.02$ & $1.07 \pm 0.01$ & $1.30 \pm 0.02$ & $1.45 \pm 0.04$ & $1.50 \pm 0.01$ & $1.54 \pm 0.01$ & $1.63 \pm 0.03$ \\
\hline$\sigma$ & 0.05 & 0.04 & 0.05 & 0.11 & 0.01 & 0.04 & 0.09
\end{tabular}




\begin{tabular}{r|r}
\hline 20 & \multicolumn{1}{c|}{21} \\
\hline $2.49 \pm 0.02$ & $2.54 \pm 0.04$ \\
\hline 0.09 & 0.11
\end{tabular}

Fig. 1

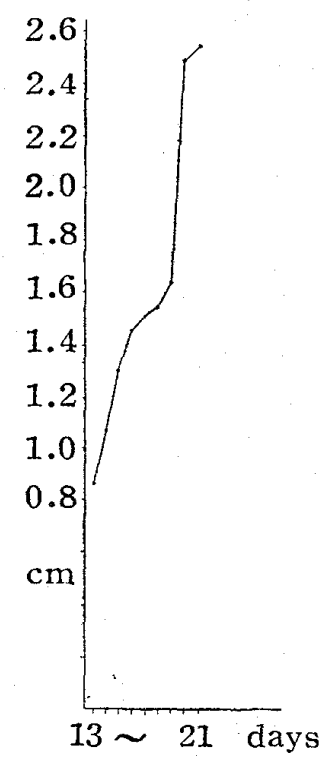

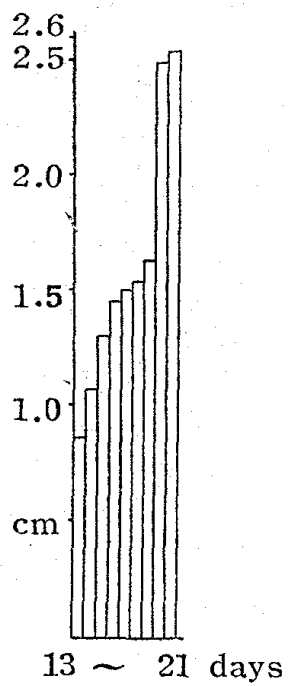

2) Body weight of the embryo.

It increases steadily from the 13 th to the 21 th day just before birth. But it increases abruptly in two days from the 19 th to the 20 th day and acquires as much as 2 times its previous weight. (Table 2, Fig. 2)

Table 2

\begin{tabular}{r|r|r|r|r|r|r|r|r|r|}
\hline Days & \multicolumn{1}{c|}{13} & \multicolumn{1}{c}{14} & 15 & 16 & 17 & 18 & 19 \\
\hline $\mathrm{M} \pm \mathrm{m}(\mathrm{gr})$ & $0.11 \pm 0.03$ & $0.26 \pm 0.01$ & $0.30 \pm 0.01$ & $0.41 \pm 0.01$ & $0.49 \pm 0.01$ & $0.56 \pm 0.01$ & $0.74 \pm 0.03$ \\
\hline$\sigma$ & 0.01 & 0.04 & 0.02 & 0.03 & 0.01 & 0.04 & 0.09
\end{tabular}

\begin{tabular}{r|r}
\hline \multicolumn{1}{c|}{20} & \multicolumn{1}{c}{21} \\
\hline $1.85 \pm 0.05$ & $1.98 \pm 0.05$ \\
\hline 0.18 & 0.15
\end{tabular}

3) Head to tail length (post-natal).

The length of $5.85 \mathrm{~cm}$ at the first day of birth increases with a constant rate attaining about 2 times increase by the 16th, about 3 times increase by the 35 th and about 4 times increase by the 50 th post-natal day. (Table 3 , Fig. 3 ).

Table 3

\begin{tabular}{|c|c|c|c|c|c|c|c|}
\hline Days & 1 & 2 & 3 & 4 & 5 & 6 & 7 \\
\hline $\mathrm{M} \pm \mathrm{m}(\mathrm{cm})$ & $5.85 \pm 0.04$ & $6.73 \pm 0.20$ & $6.95 \pm 0.05$ & $7.05 \pm 0.06$ & $7.35 \pm 0.10$ & $7.78 \pm 0.13$ & $7.98 \pm 0.11$ \\
\hline$\sigma$ & 0.09 & 0.40 & 0.10 & 0.11 & 0.20 & 0.26 & 0.22 \\
\hline
\end{tabular}




\begin{tabular}{r|r|r|r|r|r|r|r}
\hline \multicolumn{1}{c|}{8} & \multicolumn{1}{c|}{9} & 10 & \multicolumn{1}{c}{11} & 12 & 13 & 14 & 15 \\
\hline $8.08 \pm 0.15$ & $9.03 \pm 0.17$ & $9.60 \pm 0.18$ & $9.65 \pm 0.30$ & $10.65 \pm 0.34$ & $10.78 \pm 0.20$ & $11.38 \pm 0.24$ & $11.78 \pm 0.07$ \\
\hline 0.30 & 0.33 & 0.37 & 0.59 & 0.67 & 0.39 & 0.48 & 0.15
\end{tabular}

\begin{tabular}{r|r|r|r|r|r|r|r}
\hline 16 & 17 & 18 & 19 & 20 & 25 & 30 & 35 \\
\hline $12.45 \pm 0.08$ & $12.88 \pm 0.17$ & $13.03 \pm 0.29$ & $13.15 \pm 0.26$ & $13.38 \pm 0.15$ & $16.63 \pm 0.15$ & $17.45 \pm 0.15$ & $18.75 \pm 0.40$ \\
\hline 0.17 & 0.34 & 0.58 & 0.52 & 0.30 & 0.30 & 0.30 & 0.80
\end{tabular}

\begin{tabular}{|c|c|c|c|}
\hline 40 & 45 & 50 & $\mathrm{ad}$ \\
\hline $21.25+0.31$ & $22.08 \pm 0.40$ & $23.03 \pm 0.51$ & $32.00 \pm 1.27$ \\
\hline 0.61 & 0.80 & 1.02 & 4.02 \\
\hline
\end{tabular}

Fig. 3
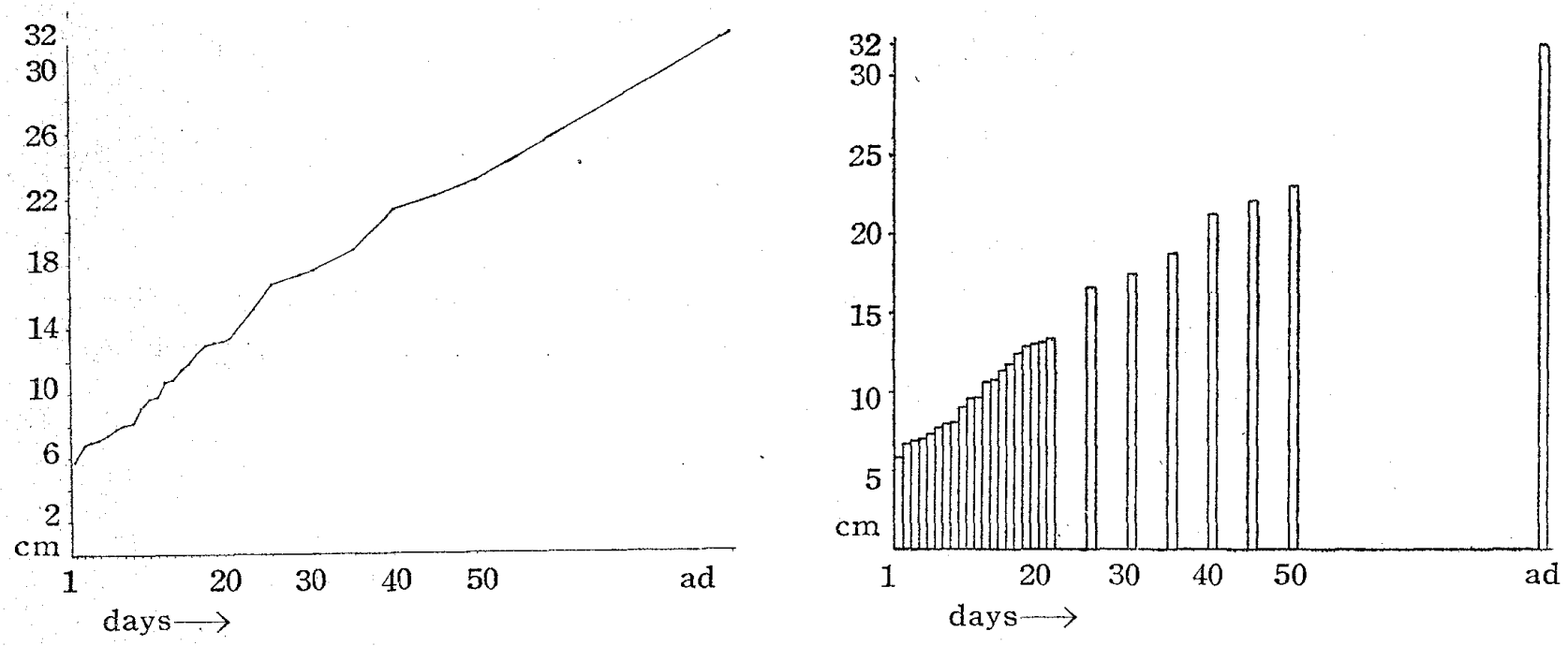

4) Body length.

The body lengths for the first, 25th and 50th day after birth were respectively 4.48 $\mathrm{cm}, 9.65 \mathrm{~cm}$, and $13.05 \mathrm{~cm}$, the rate of growth throughout the entire period remaining nearly constant. (Table 4 , Fig. 4)

Table 4

\begin{tabular}{r|r|r|r|r|r|r|r}
\hline Days & \multicolumn{1}{c|}{1} & \multicolumn{1}{c}{ 2 } & 3 & 4 & 5 & 6 & 7 \\
\hline $\mathrm{M} \pm \mathrm{m}(\mathrm{cm})$ & $4.48 \pm 0.04$ & $4.80 \pm 0.06$ & $4.73 \pm 0.13$ & $5.13 \pm 0.04$ & $5.30 \pm 0.11$ & $5.33 \pm 0.11$ & $5.75 \pm 0.10$ \\
\hline$\sigma$ & 0.08 & 0.12 & 0.25 & 0.08 & 0.22 & 0.22 & 0.21
\end{tabular}

\begin{tabular}{r|r|r|r|r|r|r|r}
\hline \multicolumn{1}{c|}{8} & \multicolumn{1}{c|}{9} & \multicolumn{1}{c|}{10} & \multicolumn{1}{c|}{11} & \multicolumn{1}{c|}{12} & 13 & 14 & 15 \\
\hline $5.88 \pm 0.07$ & $6.00 \pm 0.10$ & $6.53 \pm 0.02$ & $6.55 \pm 0.18$ & $6.93 \pm 0.16$ & $7.18 \pm 0.14$ & $7.33 \pm 0.13$ & $7.55 \pm 0.19$ \\
\hline 0.15 & 0.20 & 0.04 & 0.36 & 0.31 & 0.29 & 0.25 & 0.38
\end{tabular}


A STUDY QN THE RELATIVE GROWTH IN THE BODY OF ALBINO RATS

\begin{tabular}{r|r|r|r|r|r|r|r}
\hline \multicolumn{1}{c|}{16} & \multicolumn{1}{c|}{17} & \multicolumn{1}{c|}{18} & 19 & 20 & 25 & 30 & 35 \\
\hline $7.55 \pm 0.04$ & $7.80 \pm 0.08$ & $7.90 \pm 0.08$ & $8.43 \pm 0.13$ & $8.60 \pm 0.28$ & $9.65 \pm 0.25$ & $10.20 \pm 0.07$ & $10.30 \pm 0.35$ \\
\hline 0.09 & 0.16 & 0.16 & 0.26 & 0.56 & 0.50 & 0.14 & 0.70
\end{tabular}

\begin{tabular}{r|r|r|r}
\hline \multicolumn{1}{c|}{40} & \multicolumn{1}{c|}{45} & 50 & \multicolumn{1}{c}{ ad } \\
\hline $11.75 \pm 0.09$ & $12.03 \pm 0.13$ & $13.05 \pm 0.29$ & $19.00 \pm 0.28$ \\
\hline 0.18 & 0.26 & 0.57 & 0.89
\end{tabular}

Fig. 4
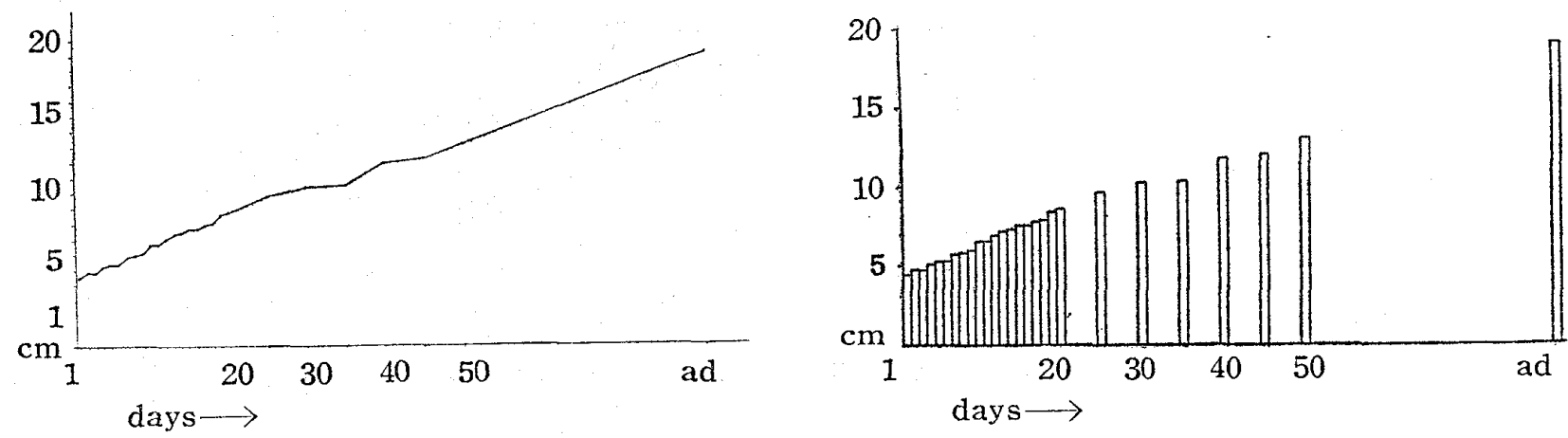

5) Body weight.

The weight $5.55 \mathrm{~g}$ shown at the first day of birth amounts 2 times that in the embryo directly before birth.

However, after that the rate of increase returns normal so that it takes for the first day value nearly 9, 15 and 19 days to attain about 2-, 3-, 4- and 12 times increases respectively. (Table 5 , Fig. 5)

Table 5

\begin{tabular}{|c|c|c|c|c|c|c|c|}
\hline Days & 1 & 2 & 3 & 4 & 5 & 6 & 7 \\
\hline$M \pm m \quad(g r)$ & $5.55 \pm 0.04$ & $5.94 \pm 0.16$ & $6.03 \pm 0.23$ & $7.05 \pm 0.28$ & $7.23 \pm 0.13$ & $8.57 \pm 0.18$ & $9.73 \pm 0.25$ \\
\hline$\sigma$ & 0.09 & 0.33 & 0.46 & 0.56 & 0.27 & 0.36 & 0.51 \\
\hline 8 & 9 & 10 & 11 & 12 & 13 & 14 & 15 \\
\hline $9.74 \pm 0.56$ & $10.84 \pm 0.95$ & $12.53 \pm 0.64$ & $14.28 \pm 0.02$ & $15.03 \pm 1.31$ & $15.38 \pm 0.98$ & $16.03 \pm 0.85$ & $16.45 \pm 0.04$ \\
\hline 1.11 & 1.90 & 1.29 & 0.04 & 2.63 & 1.96 & 1.70 & 0.09 \\
\hline 16 & 17 & 18 & 19 & 20 & 25 & 30 & 35 \\
\hline $17.60 \pm 0.04$ & $18.50 \pm 1.35$ & $18.98 \pm 0.03$ & $23.60 \pm 0.14$ & $25.95 \pm 2.07$ & $32.20 \pm 3.73$ & $32.93 \pm 0.73$ & $39.78 \div 0.59$ \\
\hline 0.08 & 2.71 & 0.06 & 0.27 & 4.14 & 7.46 & 1.46 & 1.18 \\
\hline 40 & 45 & 50 & ad & & & & \\
\hline $48.90 \pm 1.01$ & $57.23 \pm 1.53$ & $67.43 \pm 3.08$ & $197.40 \pm 11.43$ & & & . & \\
\hline 2.01 & 3.07 & 6.16 & 36.12 & & & & \\
\hline
\end{tabular}


Fig. 5
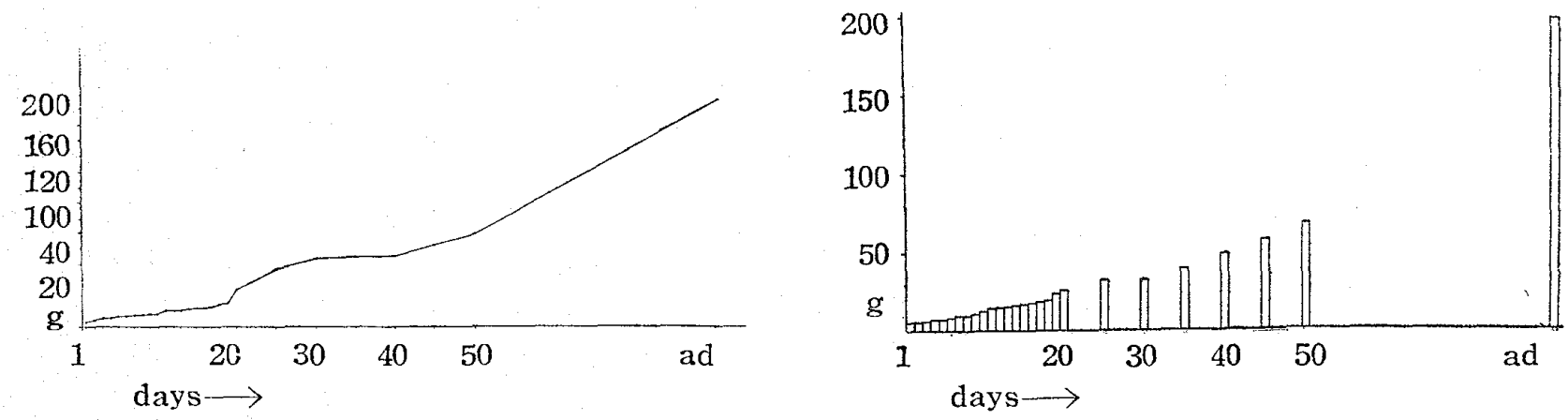

6) Length between lower lip and anus.

The value $4.08 \mathrm{~cm}$ at the first day of birth increases nearly 2 times $(8.75 \mathrm{~cm})$ by the 25 th day, nearly 3 times $(13.58 \mathrm{~cm})$ by the 50 th day showing that the rate of increase, alike that in the body length, maintains constancy thronghout the period.

(Table 6, Fig. 6)

Table 6

\begin{tabular}{|c|c|c|c|c|c|c|c|}
\hline Days & 1 & 2 & 3 & 4 & 5 & 6 & 7 \\
\hline$M \pm m(\mathrm{~cm})$ & $4.08 \pm 0.04$ & $4.23 \pm 0.07$ & $4.35 \pm 0.06$ & $4.53 \pm 0.04$ & $4.65 \pm 0.12$ & $4.65 \pm 0.19$ & $5.28 \pm 0.08$ \\
\hline$\sigma$ & 0.08 & 0.15 & 0.11 & 0.08 & 0.23 & 0.38 & 0.16 \\
\hline 8 & 9 & 10 & 11 & 12 & 13 & 14 & 15 \\
\hline $5.23 \pm 0.10$ & $5.40 \pm 0.06$ & $5.80 \pm 0$ & $5.80 \pm 0.15$ & $6.00 \pm 0.15$ & $6.33 \pm 0.28$ & $6.43 \pm 0.10$ & $6.45 \pm 0.10$ \\
\hline 0.19 & 0.12 & 0 & 0.31 & 0.31 & 0.55 & 0.19 & 0.21 \\
\hline 16 & 17 & 18 & 19 & 20 & 25 & 30 & 35 \\
\hline $6.83 \pm 0.08$ & $6.85 \pm 0.14$ & $7.00 \pm 0.10$ & $7.35 \pm 0.12$ & $7.50 \pm 0.28$ & $8.75 \pm 0.26$ & $8.98 \pm 0.22$ & $9.02 \pm 0.02$ \\
\hline 0.16 & 0.29 & 0.20 & 0.23 & 0.56 & 0.51 & 0.43 & 0.04 \\
\hline 40 & 45 & 50 & $\mathrm{ad}$ & & & & \\
\hline $10.75 \pm 0.19$ & $10.75 \pm 0.11$ & $13.58 \pm 0.23$ & $16.60 \pm 0.19$ & & & & \\
\hline 0.38 & 0.22 & 0.46 & 0.60 & & & & \\
\hline
\end{tabular}

Fig. 6
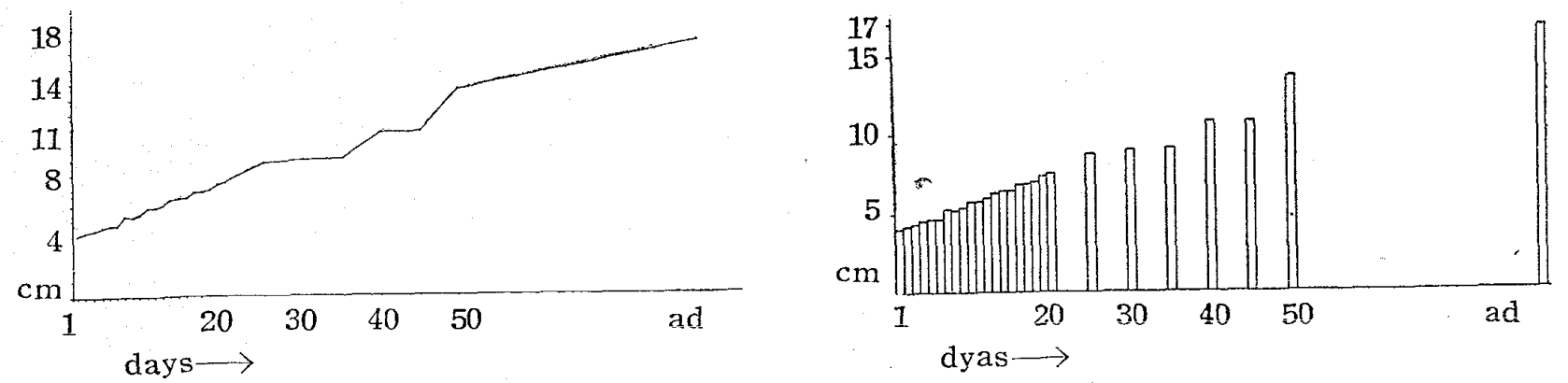

7) External orbital breadth.

The values at the $1 \mathrm{st}, 25 \mathrm{th}$ and $50 \mathrm{th}$ day after birth are respectively $1.80 \mathrm{~cm}, 1.63 \mathrm{~cm}$ 


\section{A STUDY ON THE RELATIVE GROWTH IN} THE , BODY OF ALBINO RATS

and $1.80 \mathrm{~cm}$, the rate of increase until $50 \mathrm{th}$ day being held nearly constant. The value in the adult is $1.97 \mathrm{~cm}$. (Table 7, Fig. 7)

Table 7

\begin{tabular}{|c|c|c|c|c|c|c|c|}
\hline Days & 1 & 2 & 3 & 4 & 5 & 6 & 7 \\
\hline $\mathrm{M} \pm \mathrm{m}(\mathrm{cm})$ & $1.08 \pm 0 \cdot 04$ & $1.08 \pm 0.02$ & $1.15 \pm 0.03$ & $1.18 \pm 0.02$ & $1.03 \pm 0.02$ & $1.18 \pm 0.02$ & $1.28 \div 0.02$ \\
\hline$\sigma$ & 0.08 & 0.04 & 0.05 & 0.04 & 0.04 & 0.04 & 0.04 \\
\hline 8 & 9 & 10 & 11 & 12 & 13 & 14 & 15 \\
\hline $1.34 \pm 0.02$ & $1.35 \pm 0.03$ & $1.38 \pm 0.04$ & $1.43 \pm 0.02$ & $1.43 \pm 0.02$ & $1.43 \pm 0.04$ & $1.45 \pm 0.03$ & $1.50 \pm 0.04$ \\
\hline 0.04 & 0.05 & 0.08 & 0.04 & 0.04 & 0.08 & 0.05 & 0.07 \\
\hline
\end{tabular}

\begin{tabular}{|c|c|c|c|c|c|c|c|}
\hline 16 & 17 & 18 & 19 & 20 & 25 & 30 & 35 \\
\hline $1.50 \pm 0.04$ & $1.50 \pm 0$ & $1.50 \pm 0$ & $1.53 \pm 0.02$ & $1.58 \pm 0.02$ & $1.63 \pm 0.04$ & $1.65 \pm 0.03$ & $1.68 \pm 0.04$ \\
\hline 0.07 & 0 & 0 & 0.04 & 0.04 & 0.08 & 0.05 & 0.08 \\
\hline
\end{tabular}

\begin{tabular}{r|r|r|r}
\hline \multicolumn{1}{c|}{40} & \multicolumn{1}{c|}{45} & \multicolumn{1}{c|}{50} & \multicolumn{1}{c}{ ad } \\
\hline $1.70 \pm 0$ & $1.73 \pm 0.02$ & $1.80 \pm 0$ & $1.97 \pm 0.03$ \\
\hline 0 & 0.04 & 0 & 0.09
\end{tabular}

Fig. 7
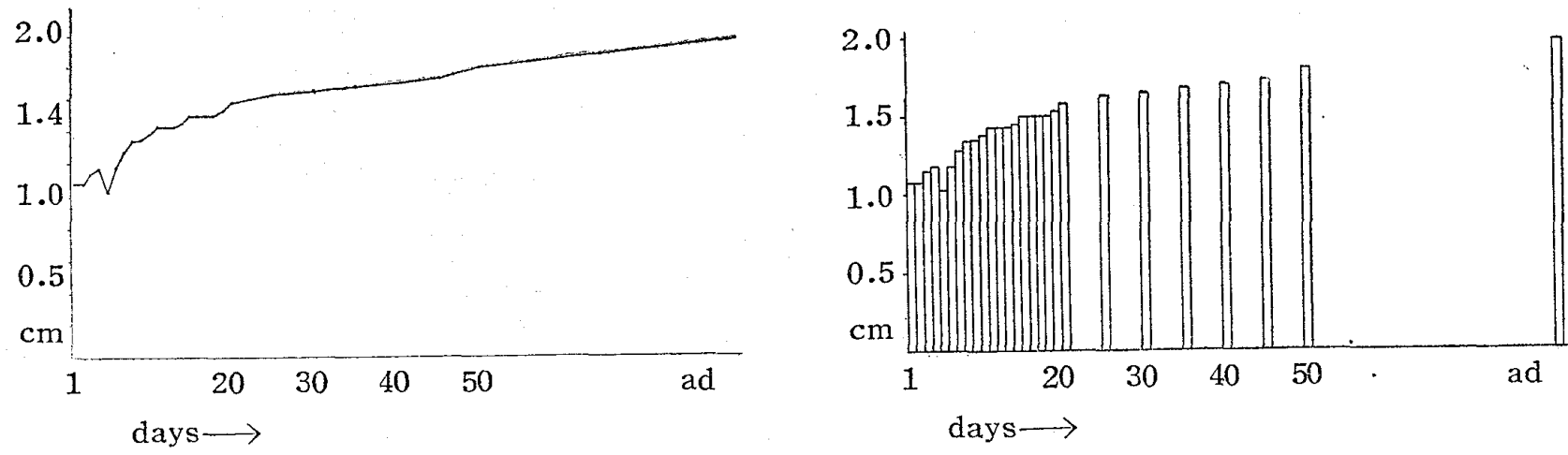

8) Internal orbital breadth.

The values at the $1 \mathrm{st}, 25 \mathrm{th}$ and 50 th day after birth are respectively $0.81 \mathrm{~cm}, 1.10 \mathrm{~cm}$ and $1.23 \mathrm{~cm}$, during which nearly the same rate of increase is maintained. As in the case of external orbital breadth even the value for the adult fails to attain twice that for the first day animal. (Table 8, Fig. 8)

Table 8

\begin{tabular}{r|r|r|r|r|r|r|r}
\hline Days & \multicolumn{1}{c|}{1} & 2 & 3 & 4 & 5 & 6 & 7 \\
\hline $\mathrm{M}_{ \pm \mathrm{m}}(\mathrm{cm})$ & $0.81 \pm 0.01$ & $0.84 \pm 0.02$ & $0.85 \pm 0.01$ & $0.86 \pm 0.01$ & $0.86 \pm 0.01$ & $0.89 \pm 0.01$ & $0.92 \pm 0.01$ \\
\hline$\sigma$ & 0.02 & 0.04 & 0.02 & 0.02 & 0.02 & 0.02 & 0.02
\end{tabular}




\begin{tabular}{r|c|c|c|c|c|c|c}
\hline Days & \multicolumn{1}{c|}{8} & 9 & 10 & 11 & 12 & 13 & 14 \\
\hline $\mathrm{M} \pm \mathrm{m}(\mathrm{cm})$ & $0.94 \pm 0.02$ & $0.96 \pm 0.02$ & $0.98 \pm 0.01$ & $1.00 \pm 0.01$ & $1.00 \pm 0.01$ & $1.01 \pm 0.02$ & $1.03 \pm 0.04$ \\
\hline$\sigma$ & 0.04 & 0.04 & 0.02 & 0.03 & 0.03 & 0.04 & 0.08
\end{tabular}

\begin{tabular}{r|r|r|r|r|r|r|r}
\hline 15 & \multicolumn{1}{c|}{16} & 17 & 18 & 19 & 20 & 25 & 30 \\
\hline $1.06 \pm 0.02$ & $1.07 \pm 0.03$ & $1.08 \pm 0.01$ & $1.08 \pm 0.03$ & $1.08 \pm 0.01$ & $1.10 \pm 0.02$ & $1.10 \pm 0.02$ & $1.13 \pm 0.02$ \\
\hline 0.04 & 0.06 & 0.02 & 0.05 & 0.02 & 0.03 & 0.04 & 0.04
\end{tabular}

\begin{tabular}{r|r|r|r|r}
\multicolumn{1}{c|}{\begin{tabular}{c} 
35 \\
\hdashline
\end{tabular}} & \multicolumn{1}{c|}{40} & \multicolumn{1}{c|}{45} & \multicolumn{1}{c}{50} & \multicolumn{1}{c}{ ad } \\
\hline $1.13 \pm 0.03$ & $1.14 \pm 0.02$ & $1.19 \pm 0.01$ & $1.23 \pm 0.02$ & $1.41 \pm 0.07$ \\
\hline 0.06 & 0.04 & 0.03 & 0.04 & 0.24
\end{tabular}

Fig. 8
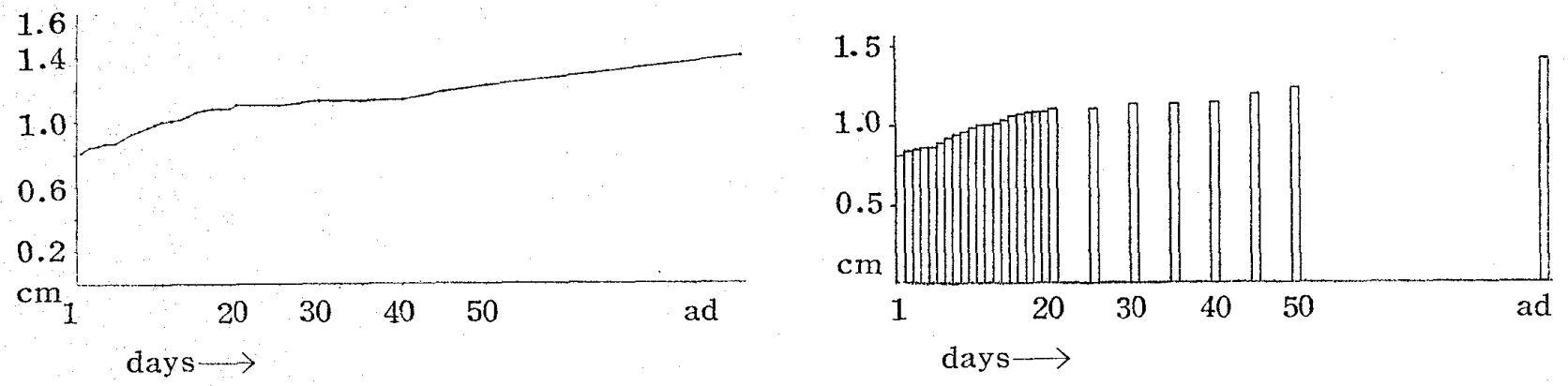

9) Breadth of rima palpebrae.

The value at the first day of birth is $0.30 \mathrm{~cm}$, thenceforth the growth until the $50 \mathrm{th}$ day being held constant.

The rate is relatively small, with the value $0.66 \mathrm{~cm}$ at the $50 \mathrm{th}$ day, nearly twice that at the lst day of birth. (Table 9, Fig. 9)

Table 9

\begin{tabular}{r|r|r|r|r|r|r|r}
\hline Days & \multicolumn{1}{c|}{1} & \multicolumn{1}{c}{2} & \multicolumn{1}{c}{3} & 4 & 5 & 6 & 7 \\
\hline $\mathrm{M} \pm \mathrm{m}(\mathrm{cm})$ & $0.30 \pm 0.01$ & $0.32 \pm 0.01$ & $0.34 \pm 0.01$ & $0.35 \pm 0.01$ & $0.37 \pm 0.01$ & $0.37 \pm 0.01$ & $0.40 \pm 0.02$ \\
\hline$\sigma$ & 0.02 & 0.02 & 0.03 & 0.03 & 0.02 & 0.02 & 0.05
\end{tabular}

\begin{tabular}{r|r|r|r|r|r|r|r|}
\multicolumn{1}{c|}{8} & \multicolumn{1}{c|}{9} & \multicolumn{1}{c|}{10} & \multicolumn{1}{c|}{11} & 12 & 13 & 14 & 15 \\
\hline $0.43 \pm 0.02$ & $0.44 \pm 0.02$ & $0.49 \pm 0.01$ & $0.49 \pm 0.01$ & $0.50 \pm 0.01$ & $0.50 \pm 0$ & $0.50 \pm 0$ & $0.50 \pm 0$ \\
\hline 0.04 & 0.04 & 0.02 & 0.02 & 0.02 & 0 & 0 & 0
\end{tabular}

\begin{tabular}{r|r|r|r|r|r|r|r}
\hline 16 & \multicolumn{1}{c|}{17} & \multicolumn{1}{c|}{18} & \multicolumn{1}{c}{19} & \multicolumn{1}{c|}{20} & 25 & 30 & 35 \\
\hline $0.50 \pm 0.02$ & $0.52 \pm 0.01$ & $0.52 \pm 0.01$ & $0.52 \pm 0.01$ & $0.56 \pm 0.02$ & $0.58 \pm 0.01$ & $0.58 \pm 0.01$ & $0.59 \pm 0.01$ \\
\hline 0.04 & 0.02 & 0.02 & 0.02 & 0.04 & 0.03 & 0.03 & 0.02
\end{tabular}


A STUDY ON THE RELATIVE GROWTH IN THE BODY OF ALBINO RATS

\begin{tabular}{r|c|c|c}
\hline \multicolumn{1}{c|c}{40} & \multicolumn{1}{c|}{45} & 50 & \multicolumn{1}{c|}{ ad } \\
\hline $0.59 \pm 0.05$ & $0.60 \pm 0.06$ & $0.66 \pm 0.01$ & $0.80 \pm 0.01$ \\
\hline 0.10 & 0.12 & 0.02 & 0.02
\end{tabular}

Fig. 9
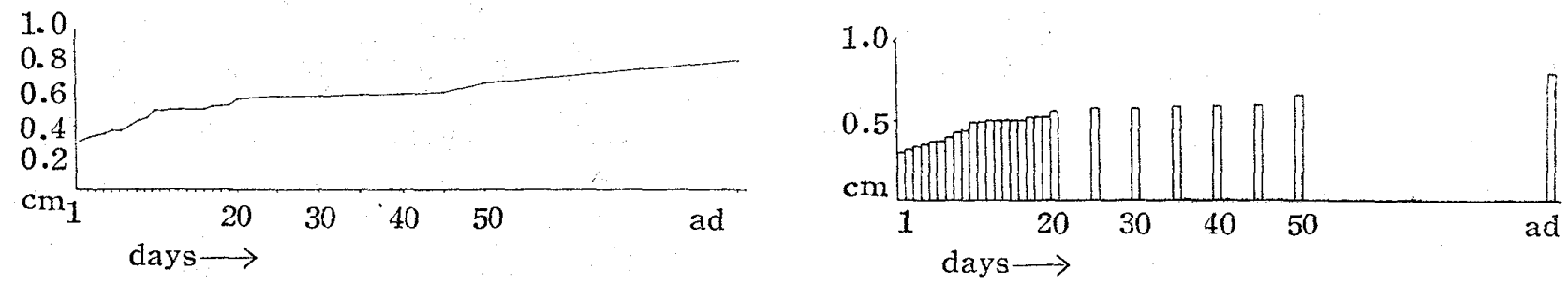

10) Mouth breadth.

The values at the Ist, $10 \mathrm{th}, 20 \mathrm{th}, 30 \mathrm{th}, 40 \mathrm{th}$ and $50 \mathrm{th}$ are respectively $0.56 \mathrm{~cm}, 0.69 \mathrm{~cm}$, $0.81 \mathrm{~cm}, 0.85 \mathrm{~cm}$ and $0.91 \mathrm{~cm}$, increasing with approximately the same constant rate. But the rate is small, the value for the adult not exceeding 1.5 times the value for Ist day animal. (Table 10, Fig.10)

Table 10

\begin{tabular}{|c|c|c|c|c|c|c|c|}
\hline Days & 1 & 2 & 3 & 4 & 5 & 6 & 7 \\
\hline$M \pm m(\mathrm{~cm})$ & $0.56 \pm 0.02$ & $0.58 \pm 0.02$ & $0.58 \pm 0.01$ & $0.60 \pm 0.002$ & $0.61 \pm 0.01$ & $0.62 \pm 0.01$ & $0.65 \pm 0.02$ \\
\hline$\sigma$ & 0.04 & 0.04 & 0.02 & 0.004 & 0.02 & 0.02 & 0.04 \\
\hline 8 & 9 & 10 & 11 & 12 & 13 & 14 & 15 \\
\hline $0.65 \pm 0.002$ & $0.68 \pm 0.01$ & $0.69 \pm 0.004$ & $0.73 \pm 0.02$ & $0.73 \pm 0.01$ & $0.74 \pm 0.03$ & $0.75 \pm 0.03$ & $0.75 \pm 0.04$ \\
\hline 0.004 & 0.02 & 0.008 & 0.04 & 0.02 & 0.05 & 0.06 & 0.09 \\
\hline 16 & 17 & 18 & 19 & 20 & 25 & 30 & 35 \\
\hline $0.77 \pm 0.02$ & $0.79 \pm 0.04$ & $0.79 \pm 0.03$ & $0.80 \pm 0.002$ & $0.81 \pm 0.02$ & $0.83 \pm 0.01$ & $0.85 \pm 0.04$ & $0.86 \pm 0.02$ \\
\hline 0.04 & 0.07 & 0.06 & 0.004 & 0.03 & 0.03 & 0.09 & 0.04 \\
\hline 40 & 45 & 50 & $\mathrm{ad}$ & & & & \\
\hline $0.88 \pm 0.02$ & $0.88 \pm 0.01$ & $0.91 \pm 0.01$ & $\overline{0.81 \pm 0.02}$ & & & & \\
\hline 0.05 & 0.02 & 0.02 & 0.05 & & & & \\
\hline
\end{tabular}

Fig. 10
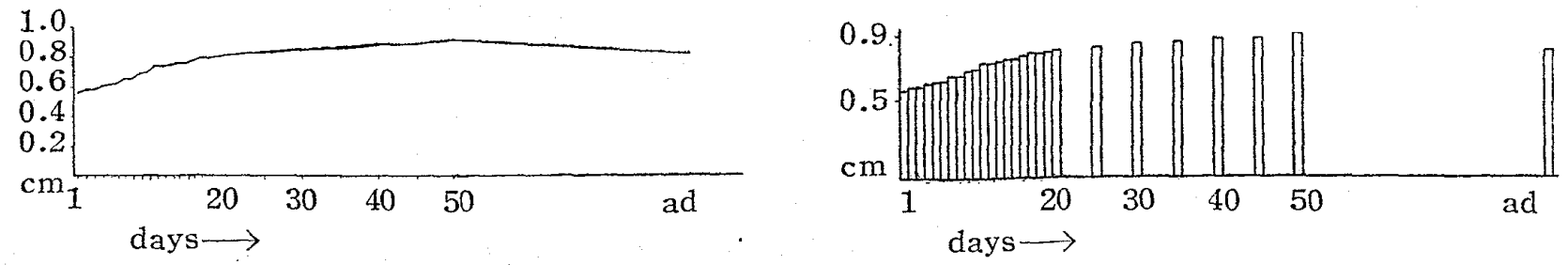

11) Maximum face breadth.

The value $0.55 \mathrm{~cm}$ at the first day of birth increases approximately twice by 11 th day 
with $1.11 \mathrm{~cm}$, but thenceforth the rate of increase becomes moderate and attains 1.49 at the 50 th day (nearly 3 times), approximately $2.10 \mathrm{~cm}$ (nearly 4 times) at the adult. (Table 11, Fig.11)

Table 11

\begin{tabular}{r|r|r|r|r|r|r|r|}
\hline \hline Days & \multicolumn{1}{c|}{1} & \multicolumn{1}{c|}{2} & 3 & 4 & 5 & 6 & 7 \\
\hline $\mathrm{M} \pm \mathrm{m}(\mathrm{cm})$ & $0.55 \pm 0.03$ & $0.61 \pm 0.01$ & $0.65 \pm 0.004$ & $0.69 \pm 0.01$ & $0.83 \pm 0.06$ & $0.87 \pm 0.01$ & $0.92 \pm 0.01$ \\
\hline$\sigma$ & 0.04 & 0.02 & 0.01 & 0.02 & 0.13 & 0.02 & 0.02 \\
\hline
\end{tabular}

\begin{tabular}{r|r|r|r|r|r|r|r}
\hline \multicolumn{1}{c|}{8} & 9 & 10 & 11 & 12 & 13 & 14 & 15 \\
\hline $0.95 \pm 0.004$ & $1.02 \pm 0.01$ & $1.06 \pm 0.004$ & $1.11 \pm 0.01$ & $1.12 \pm 0.01$ & $1.15 \pm 0.01$ & $1.16 \pm 0.01$ & $1.18 \pm 0.01$ \\
\hline 0.01 & 0.02 & 0.01 & 0.02 & 0.02 & 0.02 & 0.02 & 0.02
\end{tabular}

\begin{tabular}{r|r|r|r|r|r|r|r}
\hline 16 & 17 & 18 & 19 & 20 & 25 & 30 & 35 \\
\hline $1.20 \pm 0.03$ & $1.25 \pm 0.02$ & $1.26 \pm 0.01$ & $1.27 \pm 0.01$ & $1.31 \pm 0.01$ & $1.36 \pm 0.02$ & $1.39 \pm 0.04$ & $1.40 \pm 0.01$ \\
\hline 0.05 & 0.04 & 0.02 & 0.02 & 0.02 & 0.04 & 0.07 & 0.02
\end{tabular}

\begin{tabular}{r|r|r|r}
\hline \multicolumn{1}{c|}{40} & \multicolumn{1}{c|}{45} & \multicolumn{1}{c}{50} & \multicolumn{1}{c}{ ad } \\
\hline $1.40 \pm 0.01$ & $1.45 \pm 0.01$ & $1.49 \pm 0.02$ & $2.10 \pm 0.03$ \\
\hline 0.02 & 0.02 & 0.05 & 0.12
\end{tabular}

Fig. 11
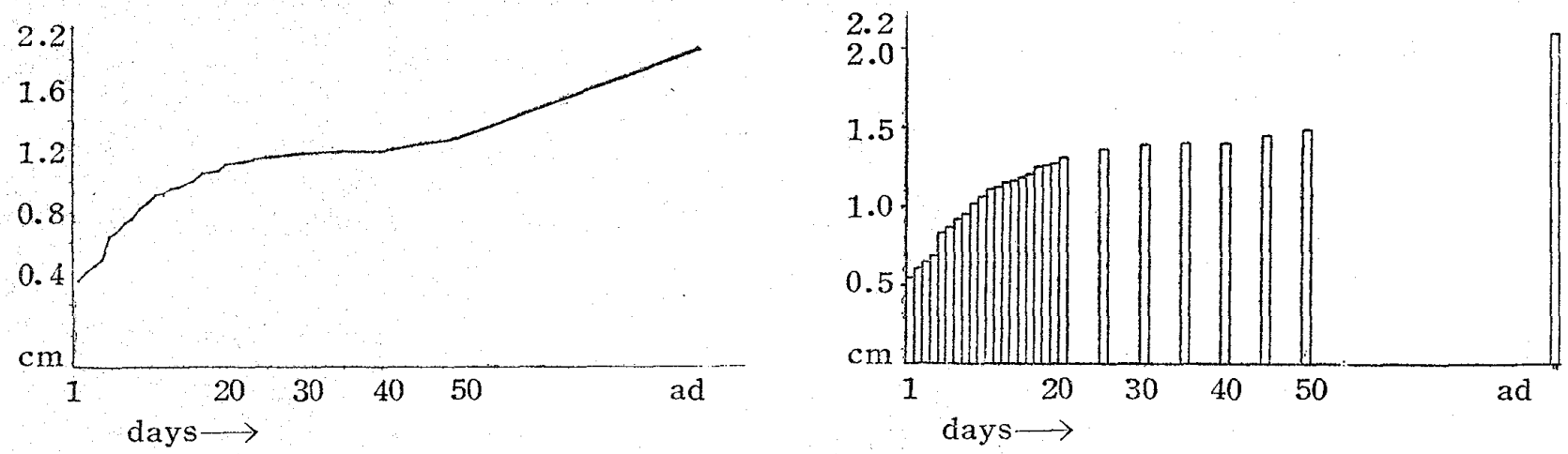

12) Maximum head length.

The value at the lst day of birth is $1.58 \mathrm{~cm}$. Thenceforth the rate of increase is moderate, showing $3.26 \mathrm{~cm}$ (nearly 2 times) by the 45 th day and $4.22 \mathrm{~cm}$ (nearly 3 times) at the adult. (Table 12, Fig. 12)

Table 12

\begin{tabular}{r|r|r|r|r|r|r|r|}
\hline Days & \multicolumn{1}{c|}{1} & 2 & 3 & 4 & 5 & 6 & 7 \\
\hline $\mathrm{M} \pm \mathrm{m}(\mathrm{cm})$ & $1.58 \pm 0.02$ & $1.60 \pm 0$ & $1.64 \pm 0.01$ & $1.70 \pm 0$ & $1.74 \pm 0.01$ & $1.82 \pm 0.01$ & $1.90 \pm 0.04$ \\
\hline$\sigma$ & 0.03 & 0 & 0.02 & 0 & 0.02 & 0.02 & 0.05 \\
\hline
\end{tabular}


A STUDY ON THE RELATIVE GROWTH IN THE BODY OF ALBINO RAIS

\begin{tabular}{r|r|r|r|r|r|r|r}
\hline \multicolumn{1}{c|}{8} & \multicolumn{1}{c|}{10} & 11 & 12 & 13 & 14 & 15 \\
\hline $1.90 \pm 0.02$ & $2.08 \pm 0.01$ & $2.26 \pm 0.02$ & $2.40 \pm 0.05$ & $2.47 \pm 0.03$ & $2.58 \pm 0.03$ & $2.65 \pm 0.01$ & $2.75 \pm 0.02$ \\
\hline 0.03 & 0.03 & 0.04 & 0.09 & 0.06 & 0.07 & 0.02 & 0.05
\end{tabular}

\begin{tabular}{r|r|r|r|r|r|r|r}
\hline 16 & 17 & 18 & 19 & 20 & 25 & 30 & 35 \\
\hline $2.79 \pm 0.04$ & $2.84 \pm 0.004$ & $2.89 \pm 0.02$ & $2.90 \pm 0.01$ & $2.91 \pm 0.01$ & $2.99 \pm 0.02$ & $3.05 \pm 0.02$ & $3.16 \pm 0.02$ \\
\hline 0.07 & 0.01 & 0.04 & 0.02 & 0.02 & 0.04 & 0.04 & 0.04
\end{tabular}

\begin{tabular}{r|c|c|c}
\hline \multicolumn{1}{c|}{40} & 45 & 50 & $\mathrm{ad}$ \\
\hline $3.20 \pm 0.01$ & $3.26 \pm 0.03$ & $3.32 \pm 0.06$ & $4.22 \pm 0.08$ \\
\hline 0.02 & 0.06 & 0.11 & 0.17
\end{tabular}

Fig. 12
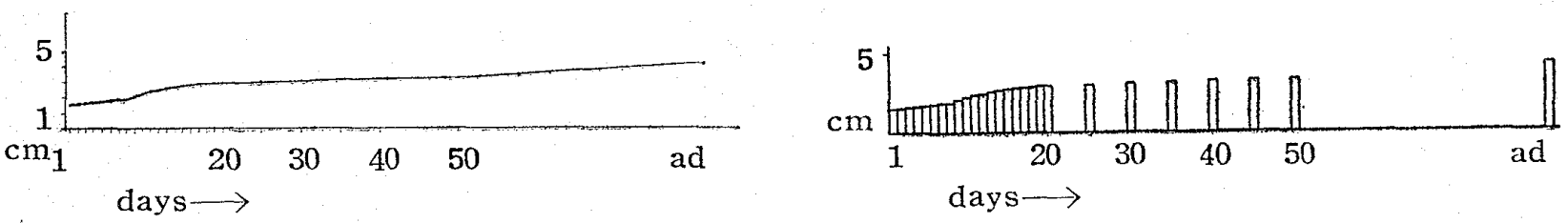

13) Maximum head breadth.

The value at the Ist day of birth is $0.81 \mathrm{~cm}$ and the value at the adult is $1.63 \mathrm{~cm}$ (nearly 2 times), showing relatively small rate of growth in comparison to other $\mathrm{p}$ arts and organs. (Table 13, Fig. 13)

Table 13

\begin{tabular}{r|r|r|r|r|r|r|r}
\hline Days & 1 & 2 & 3 & 4 & 5 & 6 & 7 \\
\hline $\mathrm{M} \pm \mathrm{m}(\mathrm{cm})$ & $0.81 \pm 0.01$ & $0.82 \pm 0$ & $0.85 \pm 0$ & $0.88 \pm 0.02$ & $0.97 \pm 0.01$ & $1.07 \pm 0.02$ & $1.08 \pm 0.02$ \\
\hline$\sigma$ & 0.02 & 0 & 0 & 0.03 & 0.02 & 0.03 & 0.03 \\
\hline 8 & 9 & 10 & 11 & 12 & 13 & 14 & 15 \\
\hline $1.07 \pm 0.01$ & $1.21 \pm 0.004$ & $1.22 \pm 0.01$ & $1.29 \pm 0.01$ & $1.29 \pm 0.01$ & $1.31 \pm 0.01$ & $1.33 \pm 0.07$ & $1.35 \pm 0.01$ \\
\hline 0.03 & 0.01 & 0.02 & 0.02 & 0.02 & 0.02 & 0.15 & 0.02 \\
\hline 16 & 17 & 18 & 19 & 20 & 25 & 30 & 35 \\
\hline $1.37 \pm 0.01$ & $1.39 \pm 0.01$ & $1.40 \pm 0.004$ & $1.41 \pm 0.01$ & $1.42 \pm 0.004$ & $1.43 \pm 0.01$ & $1.45 \pm 0.02$ & $1.4 . \pm 0.02$ \\
\hline 0.02 & 0.02 & 0.01 & 0.02 & 0.01 & 0.02 & 0.04 & 0.04 \\
\hline
\end{tabular}

\begin{tabular}{r|r|r|r}
\hline \multicolumn{1}{c|}{40} & \multicolumn{1}{c|}{45} & 50 & $\mathrm{ad}$ \\
\hline $1.46 \pm 0.01$ & $1.48 \pm 0.01$ & $1.55 \pm 0.04$ & $1.63 \pm 0.01$ \\
\hline 0.03 & 0.03 & 0.08 & 0.02
\end{tabular}


Fig. 13

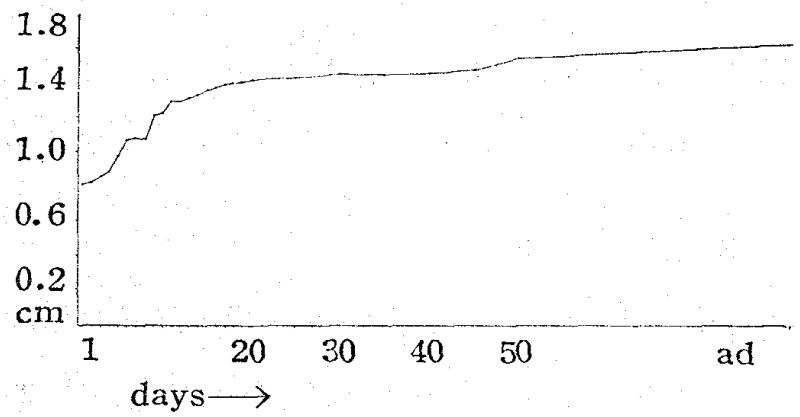

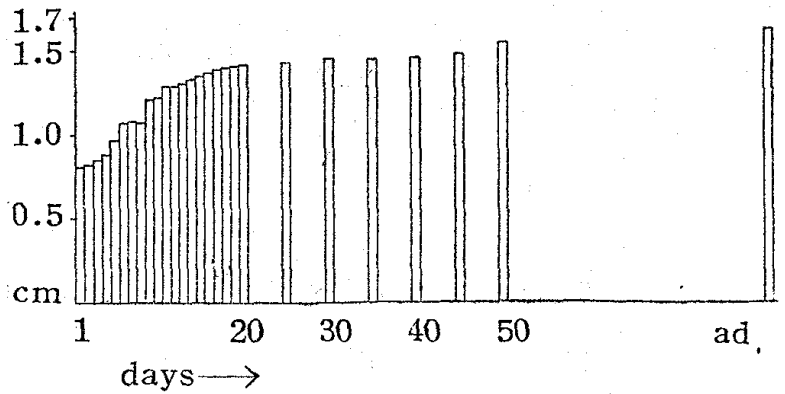

14) Length of the mandibular angle.

The measurement was done at the 8 th day of birth, when it showed $1.22 \mathrm{~cm}$ in the left, $1.29 \mathrm{~cm}$ in the right. The growth thenceforth proceeded slowly, showing in approximately 50 days $2.24 \mathrm{~cm}$ in the left $2.21 \mathrm{~cm}$ in the right, that is, only 2 times values.

This is comparatively small rate of growth. (Table 14, Fig. 14)

Fig. 14 (Right)
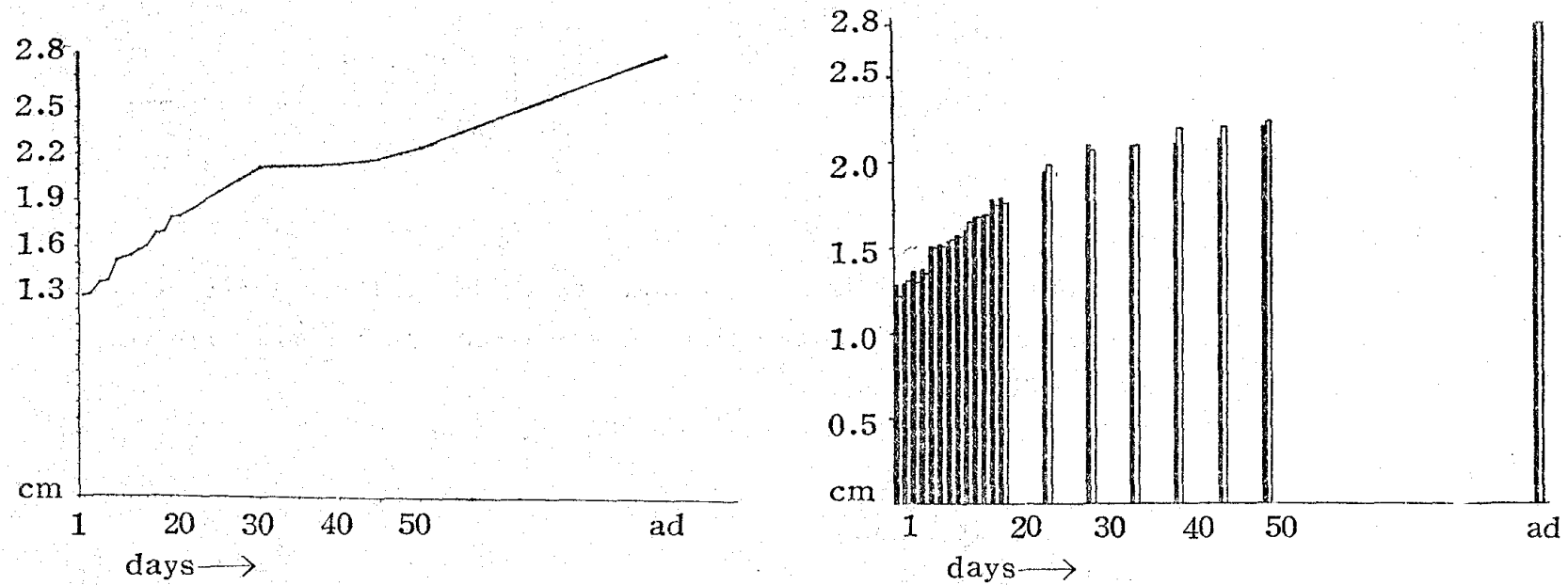

Table 14

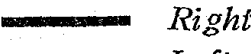

\begin{tabular}{r|r|r|r|r|r|r|r}
\hline Days & \multicolumn{1}{c|}{8} & 9 & 10 & 11 & 12 & 13 & 14 \\
\hline $\mathrm{R} \mathrm{M} \pm \mathrm{m}(\mathrm{cm})$ & $1.29 \pm 0.01$ & $1.30 \pm 0.02$ & $1.37 \pm 0.04$ & $1.38 \pm 0.04$ & $1.51 \pm 0.02$ & $1.52 \pm 0.04$ & $1.54 \pm 0.03$ \\
\hline$\sigma$ & 0.03 & 0.05 & 0.08 & 0.08 & 0.05 & 0.08 & 0.07 \\
\hline $\mathrm{L} \mathrm{M} \pm \mathrm{m}(\mathrm{cm})$ & $1.22 \pm 0.02$ & $1.31 \pm 0.04$ & $1.30 \pm 0.01$ & $1.35 \pm 0.04$ & $1.50 \pm 0.03$ & $1.51 \pm 0.003$ & $1.55 \pm 0.05$ \\
\hline$\sigma$ & 0.03 & 0.07 & 0.03 & 0.08 & 0.06 & 0.01 & 0.09
\end{tabular}

\begin{tabular}{r|r|r|r|r|r|r|r|}
\hline \multicolumn{1}{c|}{15} & \multicolumn{1}{c|}{16} & \multicolumn{1}{c|}{17} & \multicolumn{1}{c}{18} & 19 & \multicolumn{1}{c|}{20} & 25 & 30 \\
\hline $1.58 \pm 0.02$ & $1.60 \pm 0.06$ & $1.68 \pm 0.04$ & $1.69 \pm 0.01$ & $1.78 \pm 0.02$ & $1.79 \pm 0.01$ & $1.94 \pm 0.01$ & $2.10 \pm 0.01$ \\
\hline 0.03 & 0.12 & 0.08 & 0.02 & 0.04 & 0.02 & 0.02 & 0.03 \\
\hline $1.56 \pm 0.02$ & $1.65 \pm 0.02$ & $1.68 \pm 0.05$ & $1.69 \pm 0.01$ & $1.75 \pm 0.02$ & $1.76 \pm 0.01$ & $1.98 \pm 0.01$ & $2.07 \pm 0.02$ \\
\hline 0.05 & 0.05 & 0.09 & 0.03 & 0.04 & 0.02 & 0.02 & 0.04
\end{tabular}


A STUDY ON THE RELATIVE GROWTH IN THE BODY OF ALBINO RATS

\begin{tabular}{r|r|r|r|r}
\hline \multicolumn{1}{c|}{35} & \multicolumn{1}{c|}{40} & 45 & 50 & \multicolumn{1}{c|}{ ad } \\
\hline $2.10 \pm 0.02$ & $2.11 \pm 0.01$ & $2.14 \pm 0.02$ & $2.21 \pm 0.06$ & $2.80 \pm 0.04$ \\
\hline 0.03 & 0.02 & 0.04 & 0.11 & 0.14 \\
\hline $2.10 \pm 0.01$ & $2.20 \pm 0.02$ & $2.21 \pm 0.10$ & $2.24 \pm 0.03$ & $2.81 \pm 0.04$ \\
\hline 0.02 & 0.04 & 0.19 & 0.06 & 0.14
\end{tabular}

15) Height of ramus of mandibularis.

The measurement was done at the 8 th day of birth when it showed the value $0.43 \mathrm{~cm}$ in both rami. The length increased approximately 2 times $(0.88 \mathrm{~cm}$ in both rami) in 50 days. (Table 15, Fig. 15)

Table 15

\begin{tabular}{r|r|r|r|r|r|r|r}
\hline Days & 8 & 9 & 10 & 11 & 12 & 13 & 14 \\
\hline $\mathrm{R} \mathrm{M} \pm \mathrm{m}(\mathrm{cm})$ & $0.43 \pm 0.01$ & $0.46 \pm 0.03$ & $0.50 \pm 0.03$ & $0.53 \pm 0.02$ & $0.53 \pm 0.02$ & $0.56 \pm 0.04$ & $0.58 \pm 0.01$ \\
\hline$\sigma$ & 0.02 & 0.05 & 0.06 & 0.04 & 0.04 & 0.08 & 0.02 \\
\hline $\mathrm{L} \mathrm{M} \pm \mathrm{m}(\mathrm{cm})$ & $0.43 \pm 0.01$ & $0.49 \pm 0.02$ & $0.50 \pm 0$ & $0.50 \pm 0.02$ & $0.53 \pm 0.01$ & $0.57 \pm 0.02$ & $0.59 \pm 0.03$ \\
\hline$\sigma$ & 0.02 & 0.04 & 0 & 0.05 & 0.02 & 0.03 & 0.06 \\
\hline 15 & 16 & 17 & 18 & 19 & 20 & 25 & 30 \\
\hline $0.60 \pm 0.02$ & $0.61 \pm 0.004$ & $0.62 \pm 0.01$ & $0.66 \pm 0.01$ & $0.68 \pm 0.01$ & $0.72 \pm 0.06$ & $0.80 \pm 0.03$ & $0.81 \pm 0.01$ \\
\hline 0.04 & 0.01 & 0.02 & 0.02 & 0.03 & 0.12 & 0.07 & 0.02 \\
\hline $0.59 \pm 0.01$ & $0.59 \pm 0.03$ & $0.63 \pm 0.01$ & $0.65 \pm 0.02$ & $0.66 \pm 0.01$ & $0.68 \pm 0.01$ & $0.76 \pm 0.02$ & $0.78 \pm 0.03$ \\
\hline 0.03 & 0.06 & 0.02 & 0.03 & 0.02 & 0.02 & 0.04 & 0.06
\end{tabular}

\begin{tabular}{r|r|r|r|r}
\hline \multicolumn{1}{c|}{35} & \multicolumn{1}{c|}{40} & \multicolumn{1}{c|}{45} & 50 & \multicolumn{1}{c|}{ ad } \\
\hline $0.81 \pm 0.004$ & $0.81 \pm 0.004$ & $0.86 \pm 0.02$ & $0.88 \pm 0.01$ & $1.22 \pm 0.01$ \\
\hline 0.01 & 0.01 & 0.03 & 0.02 & 0.02 \\
\hline $0.81 \pm 0.004$ & $0.82 \pm 0.01$ & $0.86 \pm 0.02$ & $0.88 \pm 0.01$ & $1.22 \pm 0.01$ \\
\hline 0.01 & 0.02 & 0.04 & 0.02 & 0.02 \\
\hline
\end{tabular}

Fig. 15 (Right)
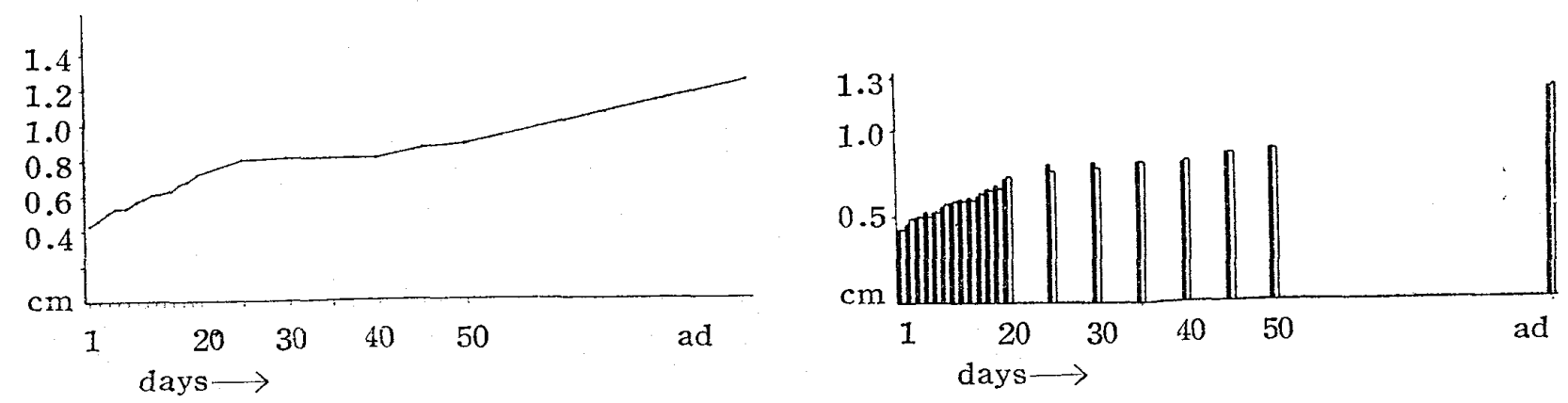
16) Length of upper extremities.

a) Humerus :

The values at the lst day of birth are $0.39 \mathrm{~cm}$ in the right, $0.40 \mathrm{~cm}$ in the left, reaching at the 15 th day $0.8 \mathrm{~cm}$ in the right and $0.82 \mathrm{~cm}$ in the left (2 times), at the $25 \mathrm{th}$ day $1.37 \mathrm{~cm}$ in both sides (nearly 3 times), at the $50 \mathrm{th}$ day $1.59 \mathrm{~cm}$ in both sides ( $\mathrm{n}$ early 4 times), and in the adult $2.39 \mathrm{~cm}$ bilaterally (nearly 6 times). The rate of growth was relatively constant. (Table 16a, Fig. 16a)

Fig. $16 \mathrm{a}$ (Right)
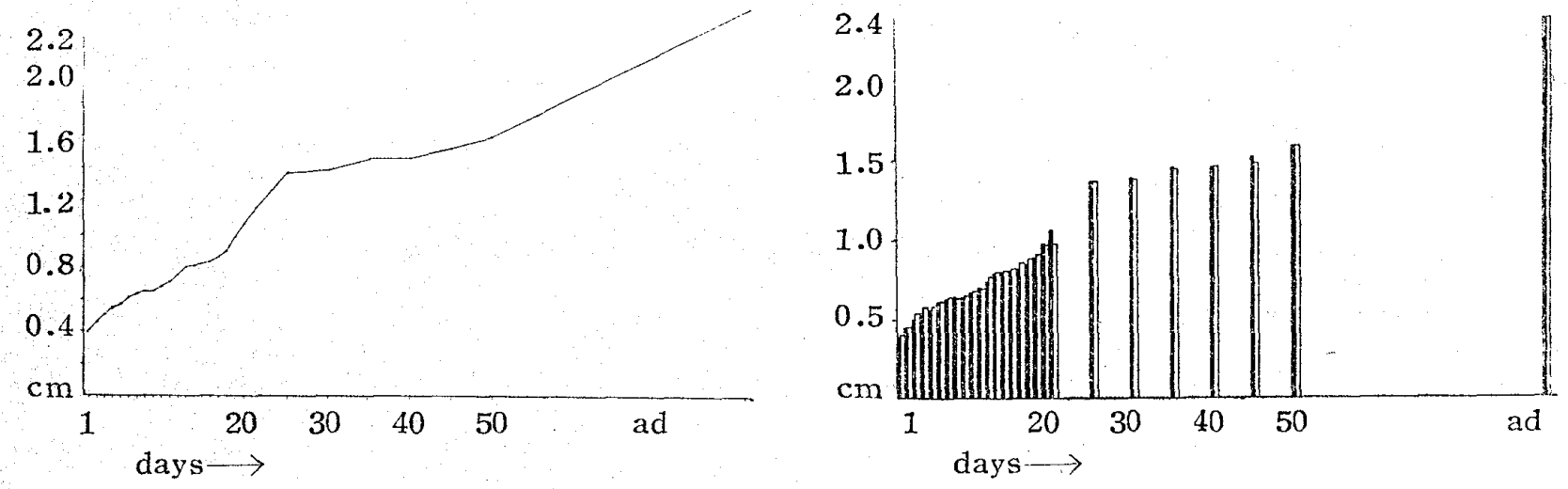

Table 16 a

\begin{tabular}{r|r|r|r|r|r|r|r}
\hline Days & 1 & 2 & 3 & 4 & 5 & 6 & 7 \\
\hline $\mathrm{R} \mathrm{M} \pm \mathrm{m}(\mathrm{cm})$ & $0.39 \pm 0.03$ & $0.45 \pm 0$ & $0.50 \pm 0.004$ & $0.54 \pm 0.01$ & $0.56 \pm 0.004$ & $0.61 \pm 0.004$ & $0.63 \pm 0.02$ \\
\hline$\sigma$ & 0.04 & 0 & 0.01 & 0.02 & 0.01 & 0.01 & 0.02 \\
\hline $\mathrm{L} \mathrm{M}_{ \pm \mathrm{m}(\mathrm{cm})}$ & $0.40 \pm 0$ & $0.45 \pm 0.02$ & $0.54 \pm 0.02$ & $0.58 \pm 0.02$ & $0.58 \pm 0.004$ & $0.61 \pm 0$ & $0.64 \pm 0.04$ \\
\hline$\sigma$ & 0 & 0.03 & 0.03 & 0.03 & 0.01 & 0 & 0.06
\end{tabular}

\begin{tabular}{r|r|r|r|r|r|r|r}
\hline \multicolumn{1}{c|}{8} & \multicolumn{1}{c|}{9} & \multicolumn{1}{c}{10} & 11 & 12 & 13 & 14 & 15 \\
\hline $0.64 \pm 0.02$ & $0.64 \pm 0.004$ & $0.67 \pm 0.01$ & $0.70 \pm 0.004$ & $0.74 \pm 0.01$ & $0.79 \pm 0.004$ & $0.80 \pm 0.04$ & $0.81 \pm 0.004$ \\
\hline 0.03 & 0.01 & 0.02 & 0.01 & 0.02 & 0.01 & 0.09 & 0.01 \\
\hline $0.63 \pm 0.02$ & $0.65 \pm 0.02$ & $0.68 \pm 0.01$ & $0.69 \pm 0.004$ & $0.77 \pm 0.01$ & $0.80 \pm 0.01$ & $0.81 \pm 0.01$ & $0.82 \pm 0.01$ \\
\hline 0.03 & 0.04 & 0.03 & 0.01 & 0.02 & 0.02 & 0.02 & 0.02
\end{tabular}

\begin{tabular}{r|r|r|r|r|r|r|r}
\hline 16 & \multicolumn{1}{|c|}{17} & \multicolumn{1}{c|}{18} & 19 & \multicolumn{1}{c|}{20} & \multicolumn{1}{c|}{25} & \multicolumn{1}{c}{30} & \multicolumn{1}{c}{35} \\
\hline $0.82 \pm 0.02$ & $0.85 \pm 0.004$ & $0.89 \pm 0.01$ & $0.98 \pm 0.06$ & $1.06 \pm 0.03$ & $1.37 \pm 0.01$ & $1.39 \pm 0.01$ & $1.46 \pm 0.02$ \\
\hline 0.04 & 0.01 & 0.02 & 0.13 & 0.02 & 0.02 & 0.03 & 0.04 \\
\hline $0.86 \pm 0.003$ & $0.88 \pm 0.03$ & $0.91 \pm 0.01$ & $0.97 \pm 0.08$ & $0.98 \pm 0.07$ & $1.37 \pm 0.01$ & $1.38 \pm 0.01$ & $1.45 \pm 0.03$ \\
\hline 0.01 & 0.06 & 0.03 & 0.16 & 0.14 & 0.02 & 0.03 & 0.05
\end{tabular}


A STUDY ON THE RELATIVE GROWTH IN THE BODY OF ALBINO RATS

\begin{tabular}{|c|c|c|c|}
\hline 40 & 45 & 50 & $\mathrm{ad}$ \\
\hline $1.46 \pm 0.02$ & $1.52 \pm 0.03$ & $1.59 \pm 0.01$ & $2.39 \pm 0.04$ \\
\hline 0.03 & 0.05 & 0.03 & 0.13 \\
\hline $1.46 \pm 0.02$ & $1.48 \pm 0.02$ & $1.59 \pm 0.01$ & $2.39 \pm 0.06$ \\
\hline 0.04 & 0.04 & 0.02 & 0.21 \\
\hline
\end{tabular}

b) Ulna :

The value at the Ist day of birth is $0.46 \mathrm{~cm}$ in the right, $0.45 \mathrm{~cm}$ in the left, at the 12 th day $0.94 \mathrm{~cm}$ in both sides (nearly 2 times), at the 50 th day $1.82 \mathrm{~cm}$ in the right, $1.79 \mathrm{~cm}$ in the left (nearly 4 times), in the adult $2.78 \mathrm{~cm}$ in the right, $2.79 \mathrm{~cm}$ in the left. (Table 16b, Fig. 16b)

Table $16 \mathrm{~b}$

\begin{tabular}{r|r|r|r|r|r|r|r}
\hline Days & 1 & 2 & 3 & 4 & 5 & 6 & 7 \\
\hline $\mathrm{R} \mathrm{M} \pm \mathrm{m}(\mathrm{cm})$ & $0.46 \pm 0.01$ & $0.53 \pm 0.02$ & $0.61 \pm 0.004$ & $0.61 \pm 0.01$ & $0.57 \pm 0$ & $0.65 \pm 0.03$ & $0.69 \pm 0.03$ \\
\hline$\sigma$ & 0.02 & 0.03 & 0.01 & 0.01 & 0 & 0.04 & 0.04 \\
\hline $\mathrm{L} \mathrm{M} \pm \mathrm{m}(\mathrm{cm})$ & $0.45 \pm 0$ & $0.49 \pm 0.01$ & $0.63 \pm 0.02$ & $0.59 \pm 0.01$ & $0.57 \pm 0.004$ & $0.66 \pm 0.03$ & $0.67 \pm 0.04$ \\
\hline$\sigma$ & 0 & 0.01 & 0.03 & 0.01 & 0.01 & 0.04 & 0.06
\end{tabular}

\begin{tabular}{r|r|r|r|r|r|r|r}
\hline \multicolumn{1}{c|}{8} & \multicolumn{1}{c|}{9} & \multicolumn{1}{c|}{10} & \multicolumn{1}{c|}{11} & 12 & 13 & 14 & 15 \\
\hline $0.69 \pm 0.04$ & $0.75 \pm 0.03$ & $0.79 \pm 0.01$ & $0.87 \pm 0.01$ & $0.94 \pm 0.02$ & $0.96 \pm 0.04$ & $1.03 \pm 0.05$ & $1.08 \pm 0.01$ \\
\hline 0.08 & 0.06 & 0.02 & 0.02 & 0.03 & 0.07 & 0.10 & 0.02 \\
\hline $0.69 \pm 0.03$ & $0.78 \pm 0.03$ & $0.78 \pm 0.03$ & $0.80 \pm 0.002$ & $0.94 \pm 0.06$ & $0.97 \pm 0.01$ & $0.99 \pm 0.004$ & $1.01 \pm 0.02$ \\
\hline 0.06 & 0.05 & 0.07 & 0.004 & 0.12 & 0.02 & 0.01 & 0.04
\end{tabular}

\begin{tabular}{r|r|r|r|r|r|r|r}
\hline \multicolumn{1}{c|}{16} & \multicolumn{1}{c|}{17} & 18 & 19 & 20 & 25 & 30 & 35 \\
\hline $1.12 \pm 0.11$ & $1.14 \pm 0.02$ & $1.16 \pm 0.01$ & $1.16 \pm 0.02$ & $1.29 \pm 0.03$ & $1.61 \pm 0.01$ & $1.63 \pm 0.02$ & $1.66 \pm 0.03$ \\
\hline 0.22 & 0.04 & 0.03 & 0.04 & 0.07 & 0.02 & 0.03 & 0.06 \\
\hline $1.04 \pm 0.01$ & $1.08 \pm 0.04$ & $1.08 \pm 0.01$ & $1.17 \pm 0.03$ & $1.17 \pm 0.02$ & $1.62 \pm 0.03$ & $1.62 \pm 0.01$ & $1.64 \pm 0.02$ \\
\hline 0.03 & 0.08 & 0.02 & 0.05 & 0.03 & 0.06 & 0.02 & 0.04
\end{tabular}

\begin{tabular}{r|r|r|r}
\hline \multicolumn{1}{c|}{40} & \multicolumn{1}{c|}{45} & 50 & $\mathrm{ad}$ \\
\hline $1.70 \pm 0.02$ & $1.74 \pm 0.04$ & $1.82 \pm 0.04$ & $2.78 \pm 0.05$ \\
\hline 0.03 & 0.08 & 0.07 & 0.17 \\
\hline $1.68 \pm 0.02$ & $1.77 \pm 0.03$ & $1.79 \pm 0.02$ & $2.79 \pm 0.04$ \\
\hline 0.04 & 0.05 & 0.03 & 0.13
\end{tabular}


Fig. $16 \mathrm{~b}$ (Right)
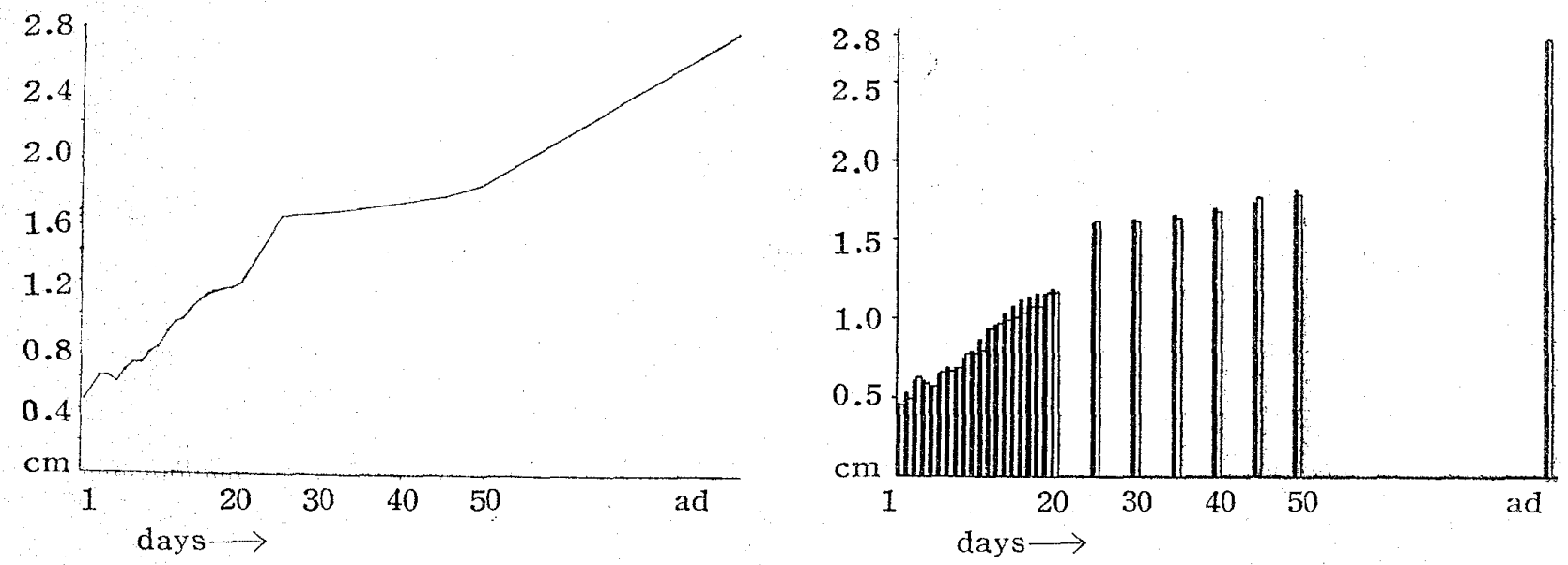

c) Radius :

The values at the lst day of birth is $0.38 \mathrm{~cm}$ bilaterally, at the 13 th day $0.77 \mathrm{~cm}$ bilaterally (nearly 2 times), at the 50 th day $1.42 \mathrm{~cm}$ in the right, $1.40 \mathrm{~cm}$ in the left (nearly 4 times), showing the same rate of growth as the preceding humerus and ulna. In the adult the value is $2.20 \mathrm{~cm}$ in the right, $2.23 \mathrm{~cm}$ in the left. (Table $16 \mathrm{c}$, Fig $16 \mathrm{c}$ )

Table $16 \mathrm{c}$

\begin{tabular}{r|r|r|r|r|r|r|r}
\hline Days & 1 & 2 & 3 & 4 & 5 & 6 & 7 \\
\hline $\mathrm{R} M \pm \mathrm{m}(\mathrm{cm})$ & $0.38 \pm 0$ & $0.42 \pm 0.01$ & $0.54 \pm 0.01$ & $0.47 \pm 0.004$ & $0.48 \pm 0.004$ & $0.51 \pm 0.01$ & $0.56 \pm 0.03$ \\
\hline$\sigma$ & 0 & 0.02 & 0.02 & 0.01 & 0.01 & 0.02 & 0.04 \\
\hline $\mathrm{L} M \pm \mathrm{m}(\mathrm{cm})$ & $0.38 \pm 0.01$ & $0.42 \pm 0$ & $0.44 \pm 0.01$ & $0.48 \pm 0.004$ & $0.48 \pm 0$ & $0.52 \pm 0.01$ & $0.56 \pm 0.03$ \\
\hline$\sigma$ & 0.02 & 0 & 0.02 & 0.01 & 0 & 0.02 & 0.04
\end{tabular}

\begin{tabular}{r|r|r|r|r|r|r|r|}
\hline 8 & \multicolumn{1}{c|}{9} & \multicolumn{1}{c|}{10} & 11 & 12 & 13 & 14 & 15 \\
\hline $0.60 \pm 0$ & $0.66 \pm 0.02$ & $0.66 \pm 0.02$ & $0.67 \pm 0.01$ & $0.74 \pm 0.01$ & $0.77 \pm 0.02$ & $0.80 \pm 0$ & $0.81 \pm 0.002$ \\
\hline 0 & 0.04 & 0.04 & 0.02 & 0.02 & 0.04 & 0 & 0.004 \\
\hline $0.61 \pm 0.01$ & $0.61 \pm 0.004$ & $0.64 \pm 0.01$ & $0.69 \pm 0.01$ & $0.75 \pm 0.002$ & $0.77 \pm 0.05$ & $0.78 \pm 0.01$ & $0.78 \pm 0.04$ \\
\hline 0.02 & 0.01 & 0.03 & 0.02 & 0.004 & 0.09 & 0.03 & 0.07
\end{tabular}

\begin{tabular}{r|r|r|r|r|r|r|r|}
\hline \multicolumn{1}{c|}{16} & \multicolumn{1}{c|}{17} & \multicolumn{1}{c|}{18} & 19 & 20 & 25 & 30 & 35 \\
\hline $0.82 \pm 0.05$ & $0.83 \pm 0.01$ & $0.89 \pm 0.01$ & $0.93 \pm 0.03$ & $0.98 \pm 0.05$ & $1.28 \pm 0.03$ & $1.30 \pm 0.02$ & $1.34 \pm 0.05$ \\
\hline 0.10 & 0.02 & 0.02 & 0.06 & 0.10 & 0.05 & 0.05 & 0.09 \\
\hline $0.79 \pm 0.02$ & $0.82 \pm 0.01$ & $0.83 \pm 0.01$ & $0.91 \pm 0.02$ & $0.91 \pm 0$ & $1.28 \pm 0.01$ & $1.34 \pm 0.03$ & $1.35 \pm 0.02$ \\
\hline 0.03 & 0.02 & 0.02 & 0.05 & 0 & 0.02 & 0.08 & 0.03
\end{tabular}




\begin{tabular}{r|r|r|r}
\hline \multicolumn{1}{c|}{40} & \multicolumn{1}{c|}{45} & \multicolumn{1}{c|}{50} & \multicolumn{1}{c}{$\mathrm{ad}$} \\
\hline $1.35 \pm 0.01$ & $1.37 \pm 0.01$ & $1.42 \pm 0.01$ & $2.20 \pm 0.04$ \\
\hline 0.02 & 0.02 & 0.02 & 0.13 \\
\hline $1.37 \pm 0.01$ & $1.38 \pm 0.01$ & $1.40 \pm 0.01$ & $2.23 \pm 0.04$ \\
\hline 0.02 & 0.02 & 0.02 & 0.13
\end{tabular}

Fig. 16c (Right)
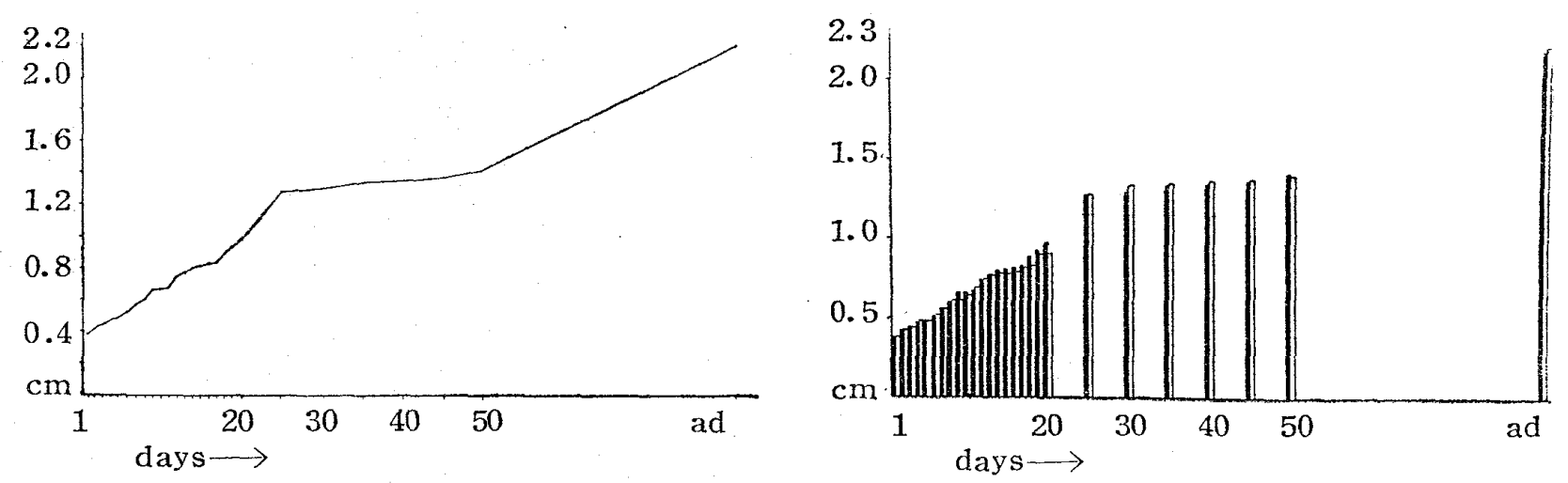

17) Length of lower extremities.

a) Femur :

The values at the lst day of birth are $0.38 \mathrm{~cm}$ in the right, $0.43 \mathrm{~cm}$ in the left, at the $14 \mathrm{th}$ day $0.84 \mathrm{~cm}$ in the right, $0.85 \mathrm{~cm}$ in the left (nearly 2 times), at the $40 \mathrm{th}$ day 1.7 $8 \mathrm{~cm}, 1.65 \mathrm{~cm}$ in the left (nearly 4 times) and at the adult $3.10 \mathrm{~cm}$ in the right, $3.16 \mathrm{~cm}$ in the left showing nearly 8 times growth. (Table 17a, Fig. 17a)

Fig. 17 a (Right)
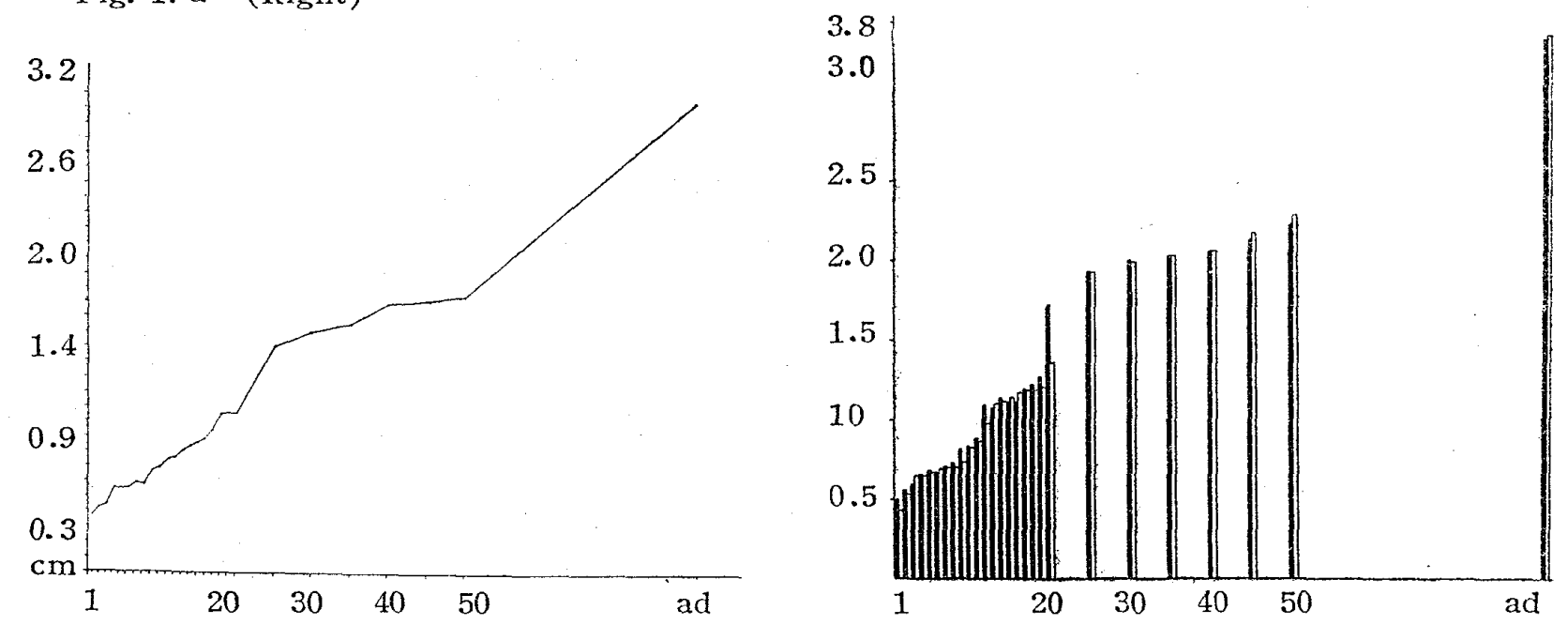

Table 17 a

\begin{tabular}{r|r|r|r|r|r|r|r}
\hline Days & 1 & 2 & 3 & 4 & 5 & 6 & 7 \\
\hline $\mathrm{R} \mathrm{M} \pm \mathrm{m}(\mathrm{cm})$ & $0.38 \pm 0$ & $0.43 \pm 0.02$ & $0.45 \pm 0$ & $0.56 \pm 0.03$ & $0.56 \pm 0.01$ & $0.56 \pm 0.01$ & $0.60 \pm 0.004$ \\
\hline$\sigma$ & 0 & 0.03 & 0 & 0.04 & 0.02 & 0.02 & 0.01 \\
\hline $\mathrm{I} M \pm \mathrm{m}(\mathrm{cm})$ & $0.43 \pm 0.02$ & $0.50 \pm 0$ & $0.55 \pm 0.04$ & $0.54 \pm 0.01$ & $0.55 \pm 0.01$ & $0.56 \pm 0.01$ & $0.60 \pm 0$ \\
\hline$\sigma$ & 0.03 & 0 & 0.06 & 0.02 & 0.02 & 0.02 & 0
\end{tabular}


A STUDY ON THE RELATIVE GROWTH IN THE BODY OF ALBINO RATS

\begin{tabular}{|c|c|c|c|c|c|c|c|}
\hline 8 & 9 & 10 & 11 & 12 & 13 & 14 & 15 \\
\hline $0.59 \pm 0.01$ & $0.68 \pm 0.01$ & $0.70 \pm 0.03$ & $0.75 \pm 0.04$ & $0.77 \pm 0.01$ & $0.81 \pm 0.02$ & $0.84 \pm 0.01$ & $0.86 \pm 0.01$ \\
\hline 0.02 & 0.03 & 0.06 & 0.08 & 0.03 & 0.04 & 0.03 & 0.02 \\
\hline $0.58 \pm 0.02$ & $0.64 \pm 0.01$ & $0.65 \pm 0.01$ & $0.76 \pm 0.03$ & $0.76 \pm 0.02$ & $0.80 \pm 0.01$ & $0.85 \pm 0.01$ & $0.88 \pm 0.01$ \\
\hline 0.04 & 0.02 & 0.02 & 0.07 & 0.04 & 0.03 & 0.02 & $\overline{0.02}$ \\
\hline 16 & 17 & 18 & 19 & 20 & 25 & 30 & 35 \\
\hline $0.89 \pm 0.04$ & $0.95 \pm 0.02$ & $1.05 \pm 0.08$ & $1.06 \pm 0.05$ & $1.06 \pm 0.02$ & $1.50 \pm 0.02$ & $1.60 \pm 0.02$ & $1.65 \pm 0.02$ \\
\hline 0.07 & 0.03 & 0.15 & 0.10 & 0.04 & 0.05 & 0.04 & 0.05 \\
\hline $0.91 \pm 0.04$ & $0.96 \pm 0.02$ & $1.05 \pm 0.06$ & $1.07 \pm 0.04$ & $1.09 \pm 0.01$ & $1.50 \pm 0.01$ & $1.55 \pm 0.02$ & $1.60 \pm 0.02$ \\
\hline 0.07 & 0.04 & 0.12 & 0.08 & 0.02 & 0.03 & 0.04 & 0.04 \\
\hline 40 & 45 & $\cdot 50$ & ad & & & & \\
\hline $1.78 \pm 0.02$ & $1.79 \pm 0.04$ & $1.82 \pm 0.21$ & $3.10 \pm 0.07$ & & & & \\
\hline 0.04 & 0.08 & 0.42 & 0.23 & & & & \\
\hline $1.65 \pm 0.03$ & $1.85 \pm 0.01$ & $1.88 \pm 0.05$ & $3.11 \pm 0.04$ & & & & \\
\hline 0.05 & 0.03 & 0.10 & 0.13 & & & & \\
\hline
\end{tabular}

b) Tibia :

The values at the lst day of birth are $0.50 \mathrm{~cm}$ in the right, $0.43 \mathrm{~cm}$ in the left, at the 20 th day $1.73 \mathrm{~cm}$ in the right, $1.36 \mathrm{~cm}$ in the left, at the $40 \mathrm{th}$ day $2.07 \mathrm{~cm}$ in the right and left, in the adult $3.40 \mathrm{~cm}$ in the right, $3.42 \mathrm{~cm}$ in the left. Compared to the value at the Ist day, the right values in every remaining stage of growth are slightly larger than the left values both eventually attaining nearly 8 times increase.

(Table17b, Fig. 17b)

Table $17 \mathrm{~b}$

\begin{tabular}{r|r|r|r|r|r|r|r}
\hline Days & 1 & 2 & 3 & 4 & 5 & 6 & 7 \\
\hline $\mathrm{R} M \pm \mathrm{m}(\mathrm{cm})$ & $0.50 \pm 0$ & $0.56 \pm 0.03$ & $0.60 \pm 0.04$ & $0.66 \pm 0.01$ & $0.68 \pm 0.004$ & $0.67 \pm 0.004$ & $0.71 \pm 0.004$ \\
\hline$\sigma$ & 0 & 0.04 & 0.05 & 0.02 & 0.01 & 0.01 & 0.01 \\
\hline $\mathrm{L} \mathrm{M} \pm \mathrm{m}(\mathrm{cm})$ & $0.43 \pm 0.02$ & $0.53 \pm 0.02$ & $0.65 \pm 0.004$ & $0.65 \pm 0.01$ & $0.67 \pm 0.01$ & $0.69 \pm 0.01$ & $0.70 \pm 0.01$ \\
\hline$\sigma$ & 0.03 & 0.03 & 0.01 & 0.02 & 0.02 & 0.02 & 0.02 \\
\hline 8 & 9 & 10 & 11 & 12 & 13 & 14 & 15 \\
\hline $0.73 \pm 0.01$ & $0.82 \pm 0.03$ & $0.84 \pm 0.02$ & $0.89 \pm 0.01$ & $1.10 \pm 0.06$ & $1.08 \pm 0.04$ & $1.14 \pm 0.02$ & $1.12 \pm 0.02$ \\
\hline 0.02 & 0.07 & 0.04 & 0.02 & 0.11 & 0.08 & 0.05 & 0.05 \\
\hline $0.70 \pm 0.01$ & $0.73 \pm 0.01$ & $0.83 \pm 0.04$ & $0.87 \pm 0.02$ & $0.98 \pm 0.02$ & $1.10 \pm 0.01$ & $1.12 \pm 0.01$ & $1.14 \pm 0.03$ \\
\hline 0.02 & 0.02 & 0.08 & 0.04 & 0.03 & 0.02 & 0.02 & 0.06
\end{tabular}


A STUDY ON THE RELATIVE GROWTH IN THE BODY OF ALBINO RATS

( 19$) \quad-217-$

\begin{tabular}{r|r|r|r|r|r|r|r}
\hline \multicolumn{1}{c|}{16} & \multicolumn{1}{c|}{17} & \multicolumn{1}{c|}{18} & 19 & 20 & 25 & 30 & 35 \\
\hline $1.12 \pm 0.02$ & $1.20 \pm 0.05$ & $1.23 \pm 0.02$ & $1.28 \pm 0.02$ & $1.73 \pm 0.17$ & $1.94 \pm 0.02$ & $2.01 \pm 0.004$ & $2.04 \pm 0.02$ \\
\hline 0.05 & 0.09 & 0.04 & 0.03 & 0.31 & 0.05 & 0.01 & 0.04 \\
\hline $1.17 \pm 0.10$ & $1.19 \pm 0.01$ & $1.19 \pm 0.06$ & $1.21 \pm 0.02$ & $1.36 \pm 0.03$ & $1.93 \pm 0.02$ & $1.99 \pm 0.01$ & $2.04 \pm 0.02$ \\
\hline 0.21 & 0.02 & 0.11 & 0.04 & 0.07 & 0.04 & 0.03 & 0.04 \\
\hline
\end{tabular}

\begin{tabular}{r|r|r|r}
\hline \multicolumn{1}{c|}{40} & \multicolumn{1}{c|}{45} & \multicolumn{1}{c|}{50} & ad \\
\hline $2.07 \pm 0.04$ & $2.14 \pm 0.02$ & $2.24 \pm 0.02$ & $3.40 \pm 0.04$ \\
\hline 0.07 & 0.04 & 0.05 & 0.13 \\
\hline $2.07 \pm 0.03$ & $2.18 \pm 0.01$ & $2.29 \pm 0.03$ & $3.42 \pm 0.05$ \\
\hline 0.06 & 0.03 & 0.06 & 0.16
\end{tabular}

Fig. 17 b (Right)
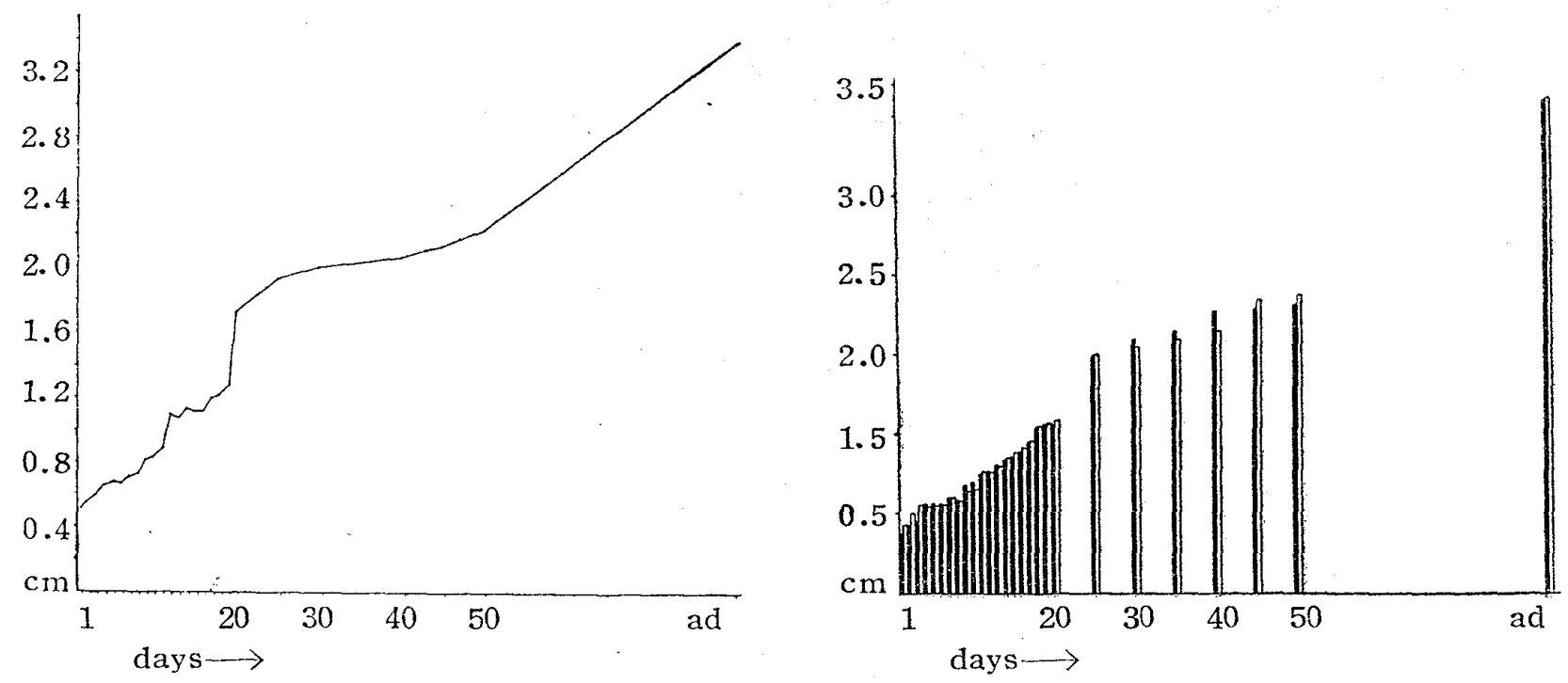

18) Length of the intestine.

The value at the first day of birth is $13.9 \mathrm{~cm}$, at the 6 th day $26.38 \mathrm{~cm}$ (nearly 2 times), at the 9 th $41.05 \mathrm{~cm}$ (nearly 3 times), at the 14 th $43.85 \mathrm{~cm}$ (nearly 4 times), showing approximately constsnt rate of growth. At the 50 th day it reaches $107.5 \mathrm{~cm}$ length (nearly 8 times) which nearly equals to that seen in the adult of the animal. (Table 18, Fig. 18)

Table 18

\begin{tabular}{r|r|r|r|r|r|r|r}
\hline Days & 1 & 2 & 3 & 4 & 5 & 6 & 7 \\
\hline $\mathrm{M} \pm \mathrm{m}(\mathrm{cm})$ & $13.93 \pm 0.75$ & $20.08 \pm 0.21$ & $23.63 \pm 0.19$ & $23.83 \pm 1.16$ & $24.85 \pm 0.75$ & $26.38 \pm 0.24$ & $29.18 \pm 0.4$ \\
\hline$\sigma$ & 1.49 & 0.42 & 0.38 & 2.31 & 1.51 & 0.49 & 0.8 \\
\hline 8 & 9 & 10 & 11 & 12 & 13 & 14 & 15 \\
\hline $33.25 \pm 3.01$ & $41.05 \pm 0.95$ & $41.65 \pm 1.17$ & $41.78 \pm 0.79$ & $42.3 \pm 1.38$ & $42.85 \pm 0.34$ & $43.85 \pm 3.12$ & $47.4 \pm 0.45$ \\
\hline 6.18 & 1.90 & 2.34 & 1.57 & 2.76 & 0.68 & 6.38 & 0.91
\end{tabular}




\begin{tabular}{l|l}
17 & 18
\end{tabular}

$18 \quad 19$

20

$+1.30$

25

$5 \quad 30$

30

\begin{tabular}{l|l|l|l|l|}
$48.53 \pm 2.57$ & $50.25 \pm 3.43$ & $50.55 \pm 2.93$ & $53.08 \pm 0.52$ & $58.03 \pm 1.30$
\end{tabular}

$61.38 \pm 1.0|64 \cdot 23 \pm 1.46| 82.25 \pm 1.44$

\begin{tabular}{lll|l|l|}
5.14 & 6.86 & 5.86 & $1.04 \mid$ & $2.61 \mid$
\end{tabular}

\subsection{8}

2.93

2.87

40 45

50
$\mathrm{ad}$

\begin{tabular}{l|l|l|l}
$84.33 \pm 1.74$ & $100.13 \pm 1.80$ & $107.5 \pm 2.36$ & $107.1 \pm 0.78$ \\
\hline
\end{tabular}

\begin{tabular}{ll|l|l}
3.49 & 3.60 & 4.72 & 2.47
\end{tabular}

Fig. 18
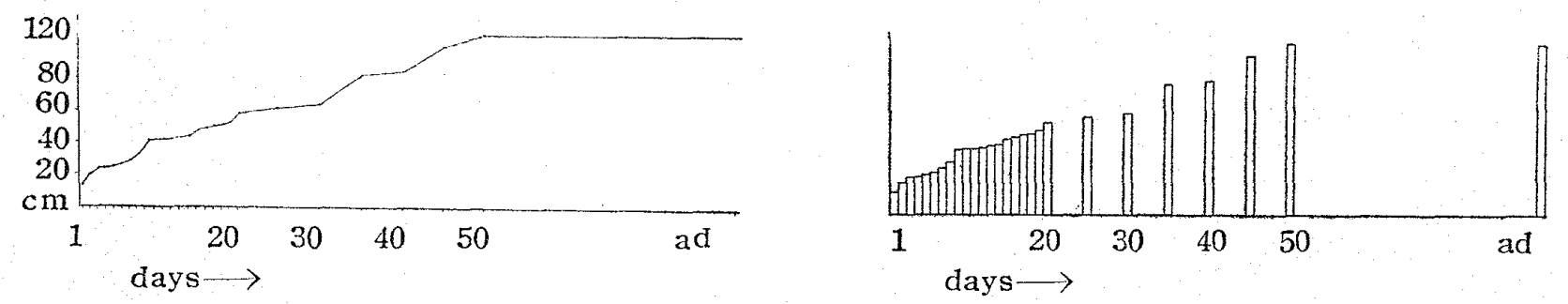

19) Heart weight.

The values at the $1 \mathrm{st}, 18 \mathrm{th}, 50 \mathrm{th}$ days and at the adult are respectively $0.015 \mathrm{~g}, 0.13 \mathrm{~g}$ (nearly 10 times), $0.525 \mathrm{~g}$ and $0.99 \mathrm{~g}$, showing approximately constant growth rate. (Table 19, Fig. 19)

Table 19

\begin{tabular}{|c|c|c|c|c|c|}
\hline Days & 1 & 2 & 3 & 4 & 5 \\
\hline$M \pm m(g r)$ & $0.015 \pm 0.001$ & $0.049 \pm 0.001$ & $0.049 \pm 0.002$ & $0.067 \pm 0.003$ & $0.075 \pm 0.001$ \\
\hline$\sigma$ & 0.002 & 0.002 & 0.003 & 0.005 & 0.002 \\
\hline 6 & 7 & 8 & 9 & 10 & 11 \\
\hline $0.078 \pm 0.008$ & $0.083 \pm 0.01$ & $0.083 \pm 0.001$ & $0.098 \pm 0.001$ & $0.099 \pm 0.001$ & $0.10 \pm 0.007$ \\
\hline 0.015 & 0.02 & 0.002 & 0.002 & 0.002 & 0.014 \\
\hline 12 & 13 & 14 & 15 & 16 & 17 \\
\hline $0.105 \pm 0.001$ & $0.105 \pm 0.004$ & $0.11 \pm 0.005$ & $0.11 \pm 0.006$ & $0.118 \pm 0.01$ & $0.123 \pm 0.01$ \\
\hline 0.002 & 0.009 & 0.01 & 0.012 & 0.02 & 0.02 \\
\hline 18 & 19 & 20 & 25 & 30 & 35 \\
\hline $0.13 \pm 0.02$ & $0.183 \pm 0.01$ & $0.195 \pm 0.01$ & $0.295 \pm 0.01$ & $0.30 \pm 0.03$ & $0.32 \pm 0.01$ \\
\hline 0.03 & 0.02 & 0.02 & 0.02 & 0.06 & 0.02 \\
\hline 40 & 45 & 50 & ad & & \\
\hline $0.463 \pm 0.01$ & $0.455 \pm 0.02$ & $0.525 \pm 0.04$ & $0.99 \pm 0.03$ & & \\
\hline 0.02 & 0.03 & 0.08 & 0.09 & & \\
\hline
\end{tabular}


Fig. 19
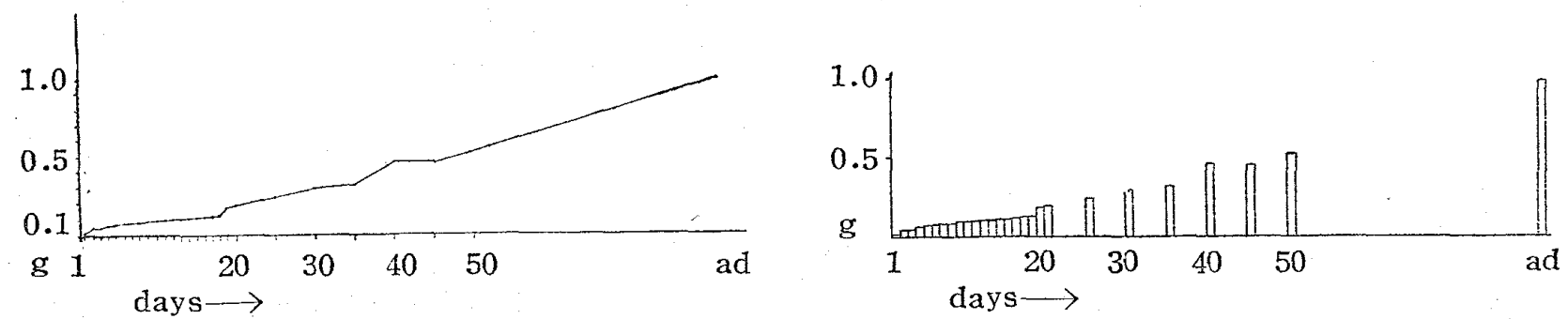

20) Lung weight.

The values at the $1 \mathrm{st}, 20 \mathrm{th}, 50 \mathrm{th}$ days and at the adult are respectively $0.13 \mathrm{~g}, 0.36 \mathrm{~g}$ (nearly 3 times), $0.79 \mathrm{~g}$ (nearly 6 times) and 1.65g. (Table 20, Fig. 20)

Table 20

\begin{tabular}{r|r|r|r|r|r|r|r}
\hline \multicolumn{1}{c|}{ Days } & \multicolumn{1}{c|}{1} & \multicolumn{1}{c}{ 2 } & 3 & 4 & 5 & 6 & 7 \\
\hline $\mathrm{M} \pm \mathrm{m}(\mathrm{gr})$ & $0.13 \pm 0.01$ & $0.15 \pm 0.002$ & $0.17 \pm 0.01$ & $0.19 \pm 0.01$ & $0.24 \pm 0.03$ & $0.24 \pm 0.01$ & $0.25 \pm 0.02$ \\
\hline$\sigma$ & 0.02 & 0.004 & 0.02 & 0.02 & 0.07 & 0.02 & 0.05
\end{tabular}

\begin{tabular}{r|c|c|c|c|c|c|c}
\hline \multicolumn{1}{c|}{8} & \multicolumn{1}{c|}{9} & \multicolumn{1}{c|}{10} & 11 & 12 & 13 & 14 & 15 \\
\hline $0.25 \pm 0.02$ & $0.25 \pm 0.03$ & $0.27 \pm 0.04$ & $0.27 \pm 0.04$ & $0.28 \pm 0.04$ & $0.29 \pm 0.03$ & $0.30 \pm 0.02$ & $0.31 \pm 0.01$ \\
\hline 0.05 & 0.05 & 0.08 & 0.08 & 0.08 & 0.07 & 0.04 & 0.02
\end{tabular}

\begin{tabular}{r|r|r|r|r|r|r|r}
\hline 16 & \multicolumn{1}{c|}{17} & \multicolumn{1}{c|}{18} & \multicolumn{1}{c|}{19} & \multicolumn{1}{c|}{20} & \multicolumn{1}{c|}{25} & 30 & 35 \\
\hline $0.32 \pm 0.02$ & $0.33 \pm 0.01$ & $0.33 \pm 0.02$ & $0.34 \pm 0.02$ & $0.36 \pm 0.02$ & $0.37 \pm 0.05$ & $0.40 \pm 0.04$ & $0.42 \pm 0.05$ \\
\hline 0.04 & 0.03 & 0.03 & 0.04 & 0.04 & 0.09 & 0.07 & 0.09
\end{tabular}

\begin{tabular}{r|r|r|r}
\hline \multicolumn{1}{c|}{40} & \multicolumn{1}{c|}{45} & \multicolumn{1}{c}{50} & \multicolumn{1}{c}{ ad } \\
\hline $0.44 \pm 0.01$ & $0.56 \pm 0.04$ & $0.79 \pm 0.03$ & $1.65 \pm 0.07$ \\
\hline 0.02 & 0.07 & 0.07 & 0.23
\end{tabular}

Fig. 20
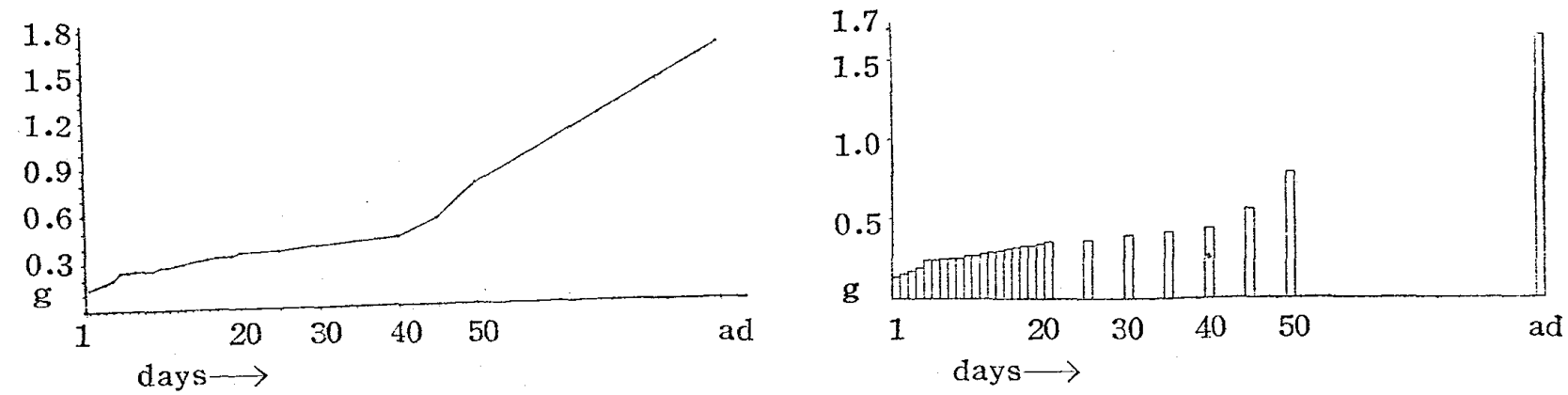


\section{A STUDY ON THE RELATIVE GROWTH IN

21) Stomach weight.

The values at the $1 \mathrm{st}, 25 \mathrm{th}$ and 50 th days of birth are respectively $0.03 \mathrm{~g}, 0.37 \mathrm{~g}$ (nearly 10 times) and $0.6 \mathrm{~g}$ (nearly 20 times) with generally constant growth rate.

(Table 21, Fig. 21)

Table 21

\begin{tabular}{|c|c|c|c|c|c|c|c|}
\hline Days & 1 & 2 & 3 & 4 & 5 & 6 & 7 \\
\hline$M+m \quad(g r)$ & $0.03 \pm 0.002$ & $0.04 \pm 0.001$ & $0.05 \pm 0.004$ & $0.05 \pm 0.001$ & $0.06 \pm 0.001$ & $0.06 \pm 0.003$ & $0.07 \pm 0.003$ \\
\hline$\sigma$ & 0.004 & 0.002 & 0.01 & 0.002 & 0.002 & 0.01 & 0.01 \\
\hline 8 & 9 & 10 & 11 & 12 & 13 & 14 & 15 \\
\hline $0.07 \pm 0.03$ & $0.08 \pm 0.01$ & $0.08 \pm 0.01$ & $0.10 \pm 0.003$ & $0.10 \pm 0.002$ & $0.10 \pm 0.01$ & $0.11 \pm 0.02$ & $0.12 \pm 0.01$ \\
\hline 0.06 & 0.02 & 0.02 & 0.01 & 0.004 & 0.02 & 0.05 & 0.02 \\
\hline 16 & 17 & 18 & 19 & 20 & 25 & 30 & 35 \\
\hline $0.12 \pm 0.01$ & $0.13 \pm 0.02$ & $0.15 \pm 0.02$ & $0.15 \pm 0.02$ & $0.24 \pm 0.02$ & $0.37 \pm 0.01$ & $0.44 \pm 0.03$ & $0.45 \pm 0.04$ \\
\hline 0.02 & 0.03 & 0.04 & 0.04 & 0.04 & 0.02 & 0.07 & 0.09 \\
\hline 40 & 45 & 50 & $a b$ & & & & \\
\hline $0.46 \pm 0.01$ & $0.53 \pm 0.06$ & $0.60 \pm 0$ & $1.46 \pm 0.08$ & & & & \\
\hline 0.02 & 0.12 & 0 & 0.25 & & & & \\
\hline
\end{tabular}

Fig. 21
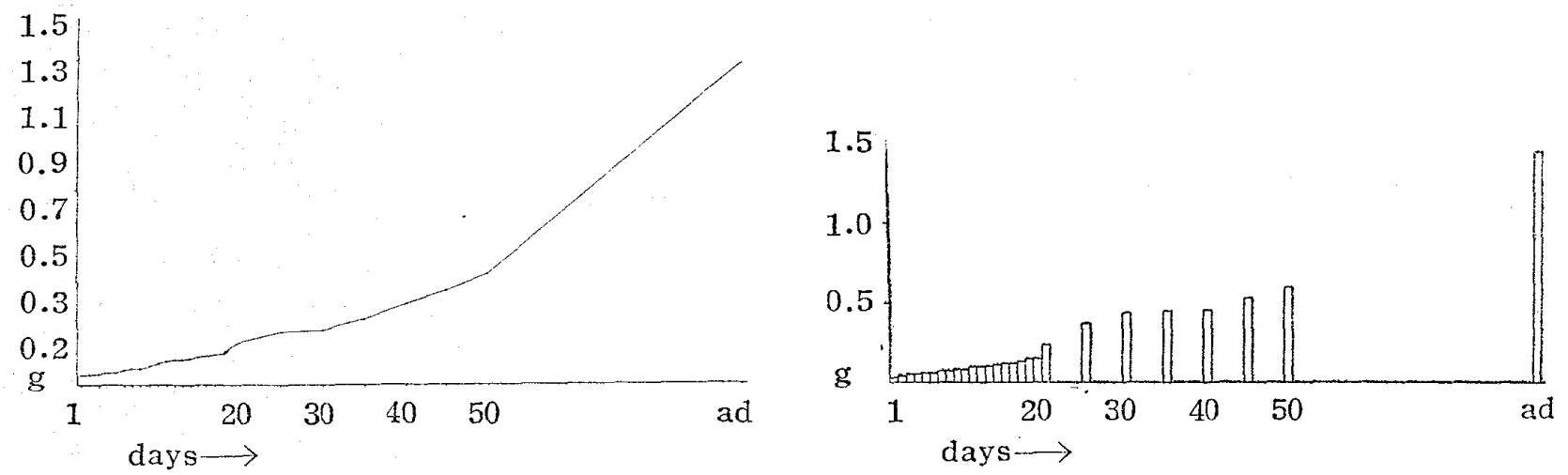

22) Liver weight.

The values at the 1 st, $11 \mathrm{th}, 16 \mathrm{th}, 20 \mathrm{th}$ and $50 \mathrm{th}$ days are respectively $0.27 \mathrm{~g}, 0.55 \mathrm{~g}(2$ times), $0.78 \mathrm{~g}$ (3 times), $1.08 \mathrm{~g}$ ( 4 times) and $4.73 \mathrm{~g}$ (18 times) with generally constant growth rate. (Table 22 , Fig. 22)

Table 22

\begin{tabular}{r|r|r|r|r|r|r|r|}
\hline Days & \multicolumn{1}{c|}{1} & \multicolumn{1}{c}{ 1 } & 3 & 4 & 5 & 6 & 7 \\
\hline $\mathrm{M} \pm \mathrm{m}(\mathrm{gr})$ & $0.27 \pm 0.02$ & $0.28 \pm 0.004$ & $0.32 \pm 0.01$ & $0.32 \pm 0.01$ & $0.35 \pm 0.004$ & $0.37 \pm 0.01$ & $0.38 \pm 0.01$ \\
\hline$\sigma$ & 0.04 & 0.01 & 0.02 & 0.02 & 0.01 & 0.02 & 0.02
\end{tabular}


A STUDY ON THE RELATIVE GROWTH IN THE BODY OF ALBINO RALS

( 23 ) $-221-$

\begin{tabular}{|c|c|c|c|c|c|c|c|}
\hline 8 & 9 & 10 & 11 & 12 & 13 & 14 & 15 \\
\hline $0.40 \pm 0.03$ & $0.44 \pm 0.02$ & $0.48 \pm 0.03$ & $0.55 \pm 0.03$ & $0.58 \pm 0.06$ & $0.58 \pm 0.05$ & $0.60 \pm 0.01$ & $0.67 \pm 0.02$ \\
\hline 0.06 & 0.01 & 0.05 & 0.06 & 0.12 & 0.09 & 0.03 & 0.03 \\
\hline 16 & 17 & 18 & 19 & 20 & 25 & 30 & 35 \\
\hline $0.78 \pm 0.02$ & $0.80 \pm 0.06$ & $0.83 \pm 0.06$ & $1.02 \pm 0.01$ & $1.08 \pm 0.03$ & $1.53 \pm 0.11$ & $1.95 \div 0.20$ & $2.14 \pm 0.19$ \\
\hline $0.0 \pm$ & 0.13 & 0.11 & 0.03 & 0.06 & 0.22 & 0.40 & 0.39 \\
\hline 40 & 45 & 50 & $a b$ & & & & \\
\hline $3.30 \pm 0.25$ & $3.95 \pm 0.14$ & $4.73 \pm 0.18$ & $10.40 \pm 0.49$ & & & & \\
\hline 0.51 & 0.27 & 0.35 & 1.56 & & & & \\
\hline
\end{tabular}

Fig. 22
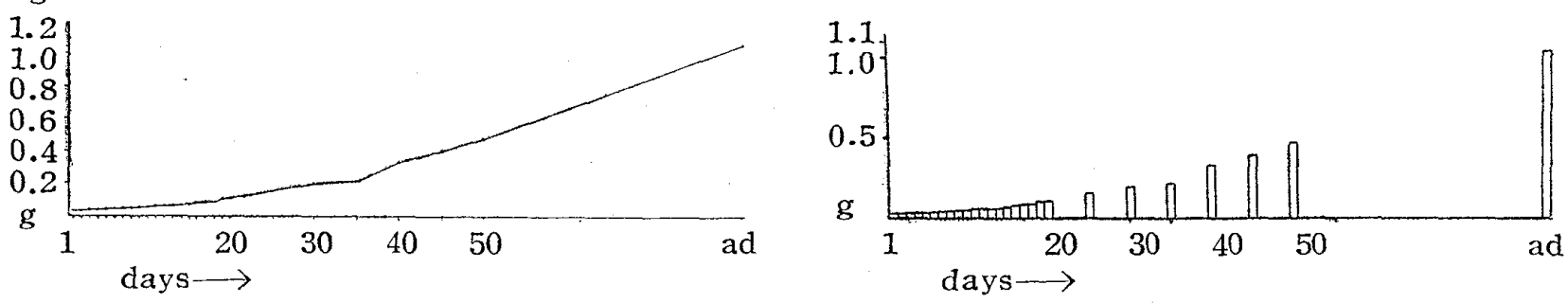

23) Kidney weight.

The value at the 1 st day of birth is $0.019 \mathrm{~g}$ which is increased 2 times $(0.045 \mathrm{~g})$ at the 5 th, nearly 4 times $(0.08 \mathrm{~g})$ at the 9 th, nearly 5 times at the $15 \mathrm{th}$, nearly 10 times at the 20th and nearly 40 times at the 50th day and thenceforth the value shows approximately $0.84 \mathrm{~g}$, nearly equivalent to those of the adult. (Table 23 , Fig. 23)

Tat le 23

\begin{tabular}{|c|c|c|c|c|c|c|c|}
\hline Days & 1 & 2 & 3 & 4 & 5 & 6 & 7 \\
\hline $\mathrm{M} \pm \mathrm{m} \quad(\mathrm{gr})$ & $0.019 \pm 0.001$ & $0.021 \pm 0.001$ & $0.030 \pm 0.001$ & $0.032 \pm 0.001$ & $0.045 \pm 0.001$ & $0.046 \pm 0.003$ & $0.049 \pm 0.003$ \\
\hline$\sigma$ & 0.002 & 0.002 & 0.002 & 0.002 & 0.002 & 0.01 & 0.01 \\
\hline 8 & 9 & 10 & 11 & 12 & 13 & 14 & 15 \\
\hline $0.050 \pm 0.004$ & $0.080 \pm 0.004$ & $0.080 \pm 0.014$ & $0.088 \pm 0.01$ & $10.09 \pm 0.01$ & $0.093 \pm 0.004$ & $0.094 \pm 0.01$ & $0.10 \div 0$ \\
\hline 0.01 & 0.01 & 0.03 & 0.02 & 0.02 & 0.01 & 0.02 & 0 \\
\hline 16 & 17 & 18 & 19 & 20 & 25 & 30 & 35 \\
\hline $0.108 \pm 0.01$ & $0.12 \pm 0.02$ & $0.145 \pm 0.01$ & $0.17 \pm 0.02$ & $0.175 \pm 0.02$ & $0.2 \pm 0$ & $0.208 \pm 0.004$ & $0.255 \div 0.02$ \\
\hline 0.02 & 0.03 & 0.02 & 0.04 & 0.04 & 0 & 0.01 & 0.04 \\
\hline 40 & 45 & 50 & ad & & & & \\
\hline $0.36 \pm 0.02$ & $0.523 \pm 0.06$ & $0.80 \pm 0.43$ & $0.84 \pm 0.03$ & & & & \\
\hline 0.03 & 0.13 & 0.87 & 0.08 & & & & \\
\hline
\end{tabular}


Fig. 23
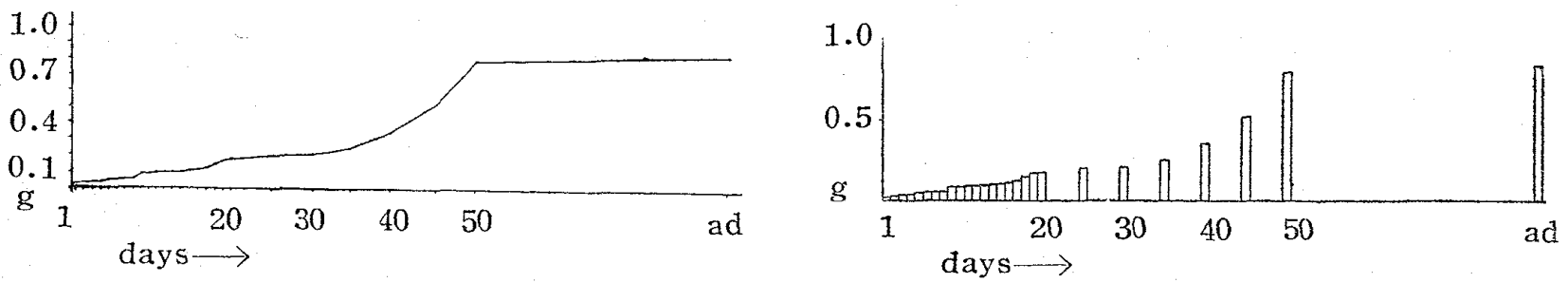

24) Spleen weight.

The value $0.008 \mathrm{~g}$ at the $1 \mathrm{st}$ day of birth is increased 10 times $(0.08 \mathrm{~g})$ at the $15 \mathrm{th}$ day, 23 times $(0.185 \mathrm{~g})$ at the 50 th day and to $0.58 \mathrm{~g}$ at the adult. (Table 24, Fig. 24)

Table 24

\begin{tabular}{|c|c|c|c|c|c|c|}
\hline Days & 1 & 2 & 3 & 4 & 5 & 6 \\
\hline $\mathrm{M} \pm \mathrm{m}$ (gr) & $0.008 \pm 0.006$ & $0.013 \pm 0.001$ & $0.013 \pm 0.0004$ & $0.017 \pm 0.0015$ & $0.020 \pm 0.0009$ & $0.022 \pm 0.0007$ \\
\hline$\sigma$ & 0.0012 & 0.002 & 0.0007 & 0.0031 & 0.0019 & 0.0014 \\
\hline 7 & 8 & 9 & 10 & 11 & 12 & 13 \\
\hline $0.031 \pm 0.007$ & $0.033 \pm 0.008$ & $0.037 \pm 0.004$ & $0.043 \pm 0.007$ & $0.043 \pm 0.006$ & $0.068 \pm 0.0004$ & $0.07 \pm 0.004$ \\
\hline 0.014 & 0.016 & 0.007 & 0.013 & 0.011 & 0.0008 & 0.007 \\
\hline 14 & 15 & 16 & 17 & 18 & 19 & 20 \\
\hline $0.075 \pm 0.006$ & $0.080 \pm 0.004$ & $0.084 \pm 0.003$ & $0.088 \pm 0.004$ & $0.088 \pm 0.011$ & $0.093 \pm 0.004$ & $0.095 \pm 0.004$ \\
\hline 0.011 & 0.007 & 0.007 & 0.008 & 0.022 & 0.008 & 0.009 \\
\hline 25 & 30 & 35 & 40 & 45 & 50 & ad \\
\hline $0.11 \pm 0.006$ & $0.113 \pm 0.011$ & $0.115 \pm 0.013$ & $0.12 \pm 0.024$ & $0.12 \pm 0.006$ & $0.185 \pm 0.008$ & $0.58 \pm 0.11$ \\
\hline 0.012 & 0.023 & 0.026 & 0.47 & 0.012 & 0.017 & 0.36 \\
\hline
\end{tabular}

Fig. 24
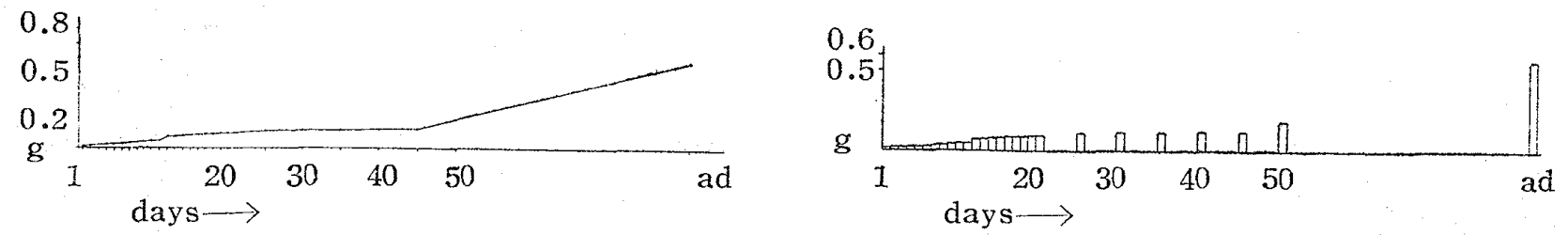

25) Thymus weight.

The value $0.005 \mathrm{~g}$ at the lst day of birth is increased 10 times $(0.053 \mathrm{~g})$ at the $16 \mathrm{th}$ day, 20 times $(0.101 \mathrm{~g})$ at the 19 th day, 40 times $(0.235 \mathrm{~g})$ at the 50 th day, showing constant growth rate. (Table 25, Fig.25)

Table 25

\begin{tabular}{|c|c|c|c|c|c|c|}
\hline Days & 1 & 2 & 3 & 4 & 5 & 6 \\
\hline$M \pm m \quad(g r)$ & $0.005 \pm 0.001$ & $0.016 \pm 0.002$ & $0.015 \pm 0.001$ & $0.018 \pm 0.001$ & $0.019 \pm 0.001$ & $0.020 \pm 0.001$ \\
\hline$\sigma$ & 0.002 & 0.004 & 0.002 & 0.002 & 0.002 & 0.002 \\
\hline
\end{tabular}


A STUDY ON THE RELATIVE GROWTH IN THE BODY OF ALBINO RATS

\begin{tabular}{r|r|r|r|r|r|r|r}
\hline \multicolumn{1}{c|}{7} & \multicolumn{1}{c|}{8} & \multicolumn{1}{c}{10} & 11 & 12 & 13 \\
\hline $0.020 \pm 0.001$ & $0.020 \pm 0.001$ & $0.021 \pm 0.001$ & $0.021 \pm 0.001$ & $0.026 \pm 0.002$ & $0.033 \pm 0.008$ & $0.033 \pm 0.005$ \\
\hline 0.002 & 0.002 & 0.002 & 0.002 & 0.004 & 0.016 & 0.010 \\
\hline 14 & 15 & 16 & 17 & 18 & 19 & 20 \\
\hline $0.042 \pm 0.03$ & $0.037 \pm 0.005$ & $0.053 \pm 0.014$ & $0.058 \pm 0.011$ & $0.098 \pm 0.002$ & $0.101 \pm 0.002$ & $0.103 \pm 0.002$ \\
\hline 0.06 & 0.011 & 0.029 & 0.023 & 0.004 & 0.004 & 0.004 \\
\hline 25 & 30 & 35 & 40 & 45 & 50 & $a d$ \\
\hline $0.120 \pm 0.01$ & $0.133 \pm 0.018$ & $0.135 \pm 0.014$ & $0.15 \pm 0.021$ & $0.183 \pm 0.01$ & $0.235 \pm 0.02$ & $0.60 \pm 0.01$ \\
\hline 0.02 & 0.036 & 0.027 & 0.041 & 0.02 & 0.04 & 0.02 \\
\hline
\end{tabular}

Fig. 25
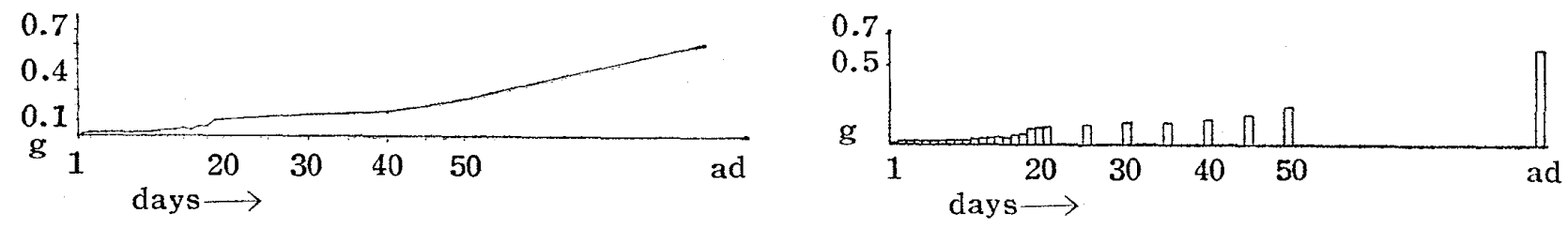

\section{THE RELATIVE GROWTH RATES}

The data from every stage of the animal's growth as presented in the foregoing were utilized in studying the relative growth of the parts of the body as well as of its internal organs in the albino rats and the values represented by $\alpha$ and log b were calculated.

Body weight against head to tail length in the embryo.

1) The relation of the body weight to the head to tail length was studied by plotting the latter on the $x$-axis and the former on the $y$-axis of logarithmic graph as shown in Fig. 26.

The relation was generally linear. When the growth of the embryo until 19th fetal day was designated as the first stadium and that at the 20 th to 21 th day as the second stadium $\alpha_{1}=2.59, \log \mathrm{b}_{1}=0.685$ and $\alpha_{2}=3.0, \log \mathrm{b}_{2}=0.124$ were respectively compuled.

However, when the relative growth of the body weight and the head to tail length were observed throughout the two stages, $\alpha=5.02$, $\log b=0.0681$ were obtained, revealing that the growth in body weight considerably exceeded that in head to tail length. (Table 26, Fig.26, Fig.40) 
Table 26

\begin{tabular}{|c|c|c|c|}
\hline$x$ & $y$ & $\alpha$ & $\log b$ \\
\hline $\begin{array}{l}\text { Head to } \\
\text { tail length }\end{array}$ & $\begin{array}{l}\text { Body weight } \\
\text { (Ist stage) }\end{array}$ & 2.59 & .6 \\
\hline " & (2nd stage) & 3.0 & 0. \\
\hline ו & (entire stages) & 5.02 & 0.0 \\
\hline Body length & Body weight & 2.42 & 0.1 \\
\hline r & $\begin{array}{l}\text { Max. } \\
\quad \text { face breadth }\end{array}$ & 0.82 & 0.2 \\
\hline If & $\begin{array}{l}\text { Max. head } \\
\text { length }\end{array}$ & 0.89 & 0.3 \\
\hline 18 & $\begin{array}{l}\text { Max. head } \\
\text { breadth }\end{array}$ & 0.52 & 0.4 \\
\hline יו & $\begin{array}{l}\text { Humerus } \\
\text { length (L) }\end{array}$ & 1.21 & $0.0^{\prime}$ \\
\hline$r$ & $\Rightarrow \quad(R)$ & 1.25 & 0.06 \\
\hline " & $\begin{array}{l}\text { Ulna } \\
\text { length (L) }\end{array}$ & 1.27 & 0.07 \\
\hline " & (R) & 1.28 & 0.07 \\
\hline r & $\begin{array}{l}\text { Radius } \\
\text { length }(L)\end{array}$ & 1.25 & 0.04 \\
\hline
\end{tabular}

\begin{tabular}{|c|c|c|c|c|}
\hline & $\mathrm{x}$ & $\mathrm{y}$ & $\alpha$ & $\operatorname{logb}$ \\
\hline Body & length & $\begin{array}{l}\text { Radius } \\
\text { length }\end{array}$ & 1.26 & 0.0629 \\
\hline " & & $\begin{array}{l}\text { Femur } \\
\text { length (L) }\end{array}$ & 1.41 & 0.0531 \\
\hline " & & $(\mathrm{R})$ & 1.48 & 0.0457 \\
\hline " & & $\begin{array}{l}\text { Tibia } \\
\text { length (L) }\end{array}$ & 1.45 & 0.0592 \\
\hline r & & (R) & 1.58 & 0.0465 \\
\hline Body & weight & Heart weight & 0.99 & 0.0078 \\
\hline " & & Lung weight & 0.58 & 0.0593 \\
\hline " & & $\begin{array}{l}\text { Stomach } \\
\text { weight }\end{array}$ & 1.12 & 0.0056 \\
\hline r & & Liver weight & 1.12 & 0.0498 \\
\hline " & & Kidney weight & 1.15 & 0.0040 \\
\hline$"$ & & Spleen weight & 1.06 & 0.0028 \\
\hline " & & $\begin{array}{l}\text { Thy mus } \\
\text { weight }\end{array}$ & 1.24 & 0.0013 \\
\hline
\end{tabular}

Relative growth after birth

2) Relative growth of body weight.

The body lengths and the body weight were respectively plotted on the $x$-axis and $y$-axis as shown in Fig.27. It was shown that both growths proceeded in parallel throughout the entire period with the same rate. The value $\alpha=2.42$, though decreased to approximately one half the value 5.02 shown at the animal's fetal stage, still marked a considerable growth rate of the body weight as compared to the length. (Table 25, Fig.27, Fig. 40)

3) Relative growth of maximum face breadth.

As shown in Fig. 28 the maximum face breadth increased nearly in parallel with the body length, but the value $\alpha=0.82$ showed slight retardation in the growth as compared to the body length. (Teble 26, Fig. 28)

4) Relative growth of maximum head length.

Both developed with nearly the same rate, but the value $\alpha=0.89$ showed a slight retardation in the growth of maximum head length as compared to the body length. (Table 26, Fig. 29)

5) Relative growth of maximum head breadth.

As shown in Fig. 30 the value $\alpha=0.52$ showed that the growth of maximum head breadth was inferior to that of body length as it was the same with the maximum 


\section{A STUDY ON THE RELATIVE GROWTH IN THE BODY OF ALBINO RATS}

head length. (Table26, Fig. 30)

6) Relative growth in the upper extremity.

The humerus, ulna and radius in both sides develop at approximately the same rate. The $\alpha$ value of humerus are 1.21 on the left, 1.25 on the right, of ulna 1.27 on the left, 1.28 on the right, of radius 1.25 on the left, 1.26 on the right,all showing very little difference between each side. As shown in Fig. $31 \mathrm{a}, \mathrm{b}, \mathrm{c}$, they slightly exceeded the growth rate of the body length. (Table 26, Fig. 31 a, b, c, Fig. 41)

7) Relative growth of lower extremity. The constituent bones develop also at approximately the same rate. The $\alpha$ values of the femur are 1.41 in the left, 1.48 in the right, of the tibia 1.45 in the left, 1.58 in the right, showing little difference between each side. The growth rates of these bones are superior to that of body length. (Table 26, Fig. 32 a, b, Fig. 41)

8) Relative growth of heart weight.

The $\alpha$ value is 0.99 throughout the entire period, showing slight retardation in the heart weight increase against the body weight increase, but generally in parallel with the latter. (Table 26, Fig. 33, Fig. 42)

9) Relative growth of lung weight.

The value $\alpha$ is 0.58 showing marked retardation of growth as compared to the body weight. (Table 26, Fig. 34, Fig. 42)

10) Relative growth of stomach weight.

The growth rate of stomach slightly exceeds that of the body weight. The $\alpha$ value 1.12 persists throughout the entire period. (Table 26, Fig. 35, Fig. 42)

11) Relative growth of liver weight.

Alike the preceding case the growth rate of liver exceeds slightly that of the body weight, the value $\alpha$ being 1.1. The growth rate of liver was nearly constant throughout the entire period. (Table 26. Fig. 36, Fig. 42)

12) Relative growth of kidney weight.

Alike the forgoing cases of stomach and liver, kidney weight increases at nearly the constant rate. The growth rate exceeds slightly that of body weight, the value being 1.115. (Table 26, Fig. 37, Fig. 42)

13) Relative growth of spleen weight.

The rate of increase is nearly constant throughout the entire period, exceeding slightly that of body weight with the value $\alpha 1.06$. (Table 26, Fig. 38, Fig. 42)

14) Relative growth of thymus weight.

As shown in Fig. 39 the rate of increase is nearly constant throughout the entire period, exceeding slightly that of body weight with the value $\alpha 1.24$. (Table 26 , Fig. 39, Fig. 42)

\section{SUMMARY}

It would be evident from the preceding data that the rates of growth are not uniform with every part and organ of the animal's body. To summarize the whole data, however, it would be concluded that the rate of growth in the body weight during the fetal period and directly after birth is especially pronounced and that the increases in the weights of every internal organ was larger than those in the other parts of the body. 
As shown in Table 26, the relative growth rates also differ between each part and organ of the body. The largest $\alpha$ value obtaind is 5.02 in the body weight in animal's fetal period, followed by 2.42 in the body weight after birth of the animals. (Fig. 26) As for the growth rates of bones, the value $\alpha$ of the lower extremities is comparatively large showing the values 1.41 to 1.58 , while that of the upper extremities slightly falls behind with the values 1.21 to 1.28 . (Fig. 41) The bones in the head and face are poor in the $\mathrm{gr}$ owth rate in comparison to the growth rate of body length showing 0.52 to 0.89 , values smaller than 1.0. Of the relative growth rates of the internal organs, the smallest is the value 0.58 for the lung, followed by 0.99 for the heart, both showing growth lesser than that of the body weight. On the contrary, the values 1.06 for the spleen, 1.12 for the stomach and the liver, 1.15 for the kidneys, 1.24 for the thymus, these values show that all these organs slightly exceed the body weight in the growth rates.

It has been known from reviewing the works of previous investigators that Shimizu had also studied the coefficients of relative growth in albino rats utilizing the combined data accumulated by Negishi and Watanabe concerning the tail, by Tappe concerning the body weight and by Donaldson concerning the various organs.

The $\alpha$ values 2.28 to 2.29 which Shimizu has given as the relative growth coefficient of the body weight against the head to tail-root length is fairly comparable with the author's value 2.42. Unfortunately no report being available as to relative growth of any part or organ of the rat embryo, the present author's value 5.02 was here given which showed a vigorous growth rate occurring in the fetal life of the animal.

Of the relative growth rates of various internal organs against the body weight, Shim$\mathrm{izu}$, concerning the heart, has given the values from 0.77 to 1.03 regardless of sex difference of the animal, and Clark has given the value approximately of 0.8 in the mice, rabbits and dogs. These are closely comparable with the author's value 0.99. Of the lung Shimizu, in the albino rats, gave the values from 0.70 to 0.84 , Brody, in the dog, 0.85 and the present author 0.58 .

Of the stomach Shimizu gave values from 0.63 to 1.34 in the albino rats, Brody 0.72 of the stomach and the intestine in the dogs and the present author 1.12 in the albino rats showing a slight difference present between these data.

Of the liver Shimizu gave values from 0.75 to 1.81 , Brody 0.7 in the dogs and the present author 1.12, an intermediate value of Shimizu's data.

Of kidneys Shimizu gave values from 0.81 to 1.75 , while the present author gave 1.15.

Of spleen Shimizu's value were 0.93 to 1.56 and the present author's 1.06 .

As shown above, the comparison of the author's data on the relative growth rates of internal organs against the body weight with those of the previous authors proves that there is general agreement between these except minor differences observed between two or three different organs.

According to Shimizu's opinion the $\alpha$ value of the internal organs did not change appreciably after the animal's body weight reached approximate value of $35 \mathrm{~g}$, and Donaldson added further that $35 \mathrm{~g}$ of body weight was gained at the 34 th to $35 \mathrm{th}$ day after birth of the animal and that this corresponded also to the 3rd molar eruption. The present author also confirmed that the animals weighed about $35 \mathrm{~g}$ at the period from 30 th to 35 th 
day after birth and that around at the same time the 3rd molar made their appearances. In this respect it would be worth mentioning here that Outhouse and Mendell observed that the time of 3rd molar eruption was least influenced either by good or poor growth of the animal, they appeared in the time when the growth activivty of the animal had reached its prime and the rates of growth of every internal organs had become constant. He also observed that no marked developmental changes took place after the eruption of the 3rd molar. These observations were confirmed also in the present author's study.

\section{CONCLUSIONS}

The development of the embryos, sucklings and adults of the albino rats were studied at intervals either daily when it dealt with the embryos from the 13th to the day of birth and with the sucklings from the lst day of birth to the 20 th day, or once in five day when it dealt with the animals from 20 th to 50 th day and on to the aduithood.

1) The items of measurement : In the embryo head to tail length and body weight, in the post-natal animal head to tail length, body length, body weight, length between lower lip and anus, external orbital breadth, internal orbital breadth, breadth of rima palpebrae, mouth breadth, maximum face breadth, maximum head length, maximum head breadth, length of mandibular angle, height of rami mandibularis, lengths of upper and lower extremities bones, length of intestine, weights of heart, lung, stomach, liver, kidneys, spleen and thymus were respectively measured.

The results indicate that the increase in body weight is most marked in the embryonic period as well as in the period directly after birth of the animal, and that the increase is greater in the internal organs than in other parts of the body.

2) The greatest $\alpha$ value 5.02 is shown in body weight against head to length in the embryonic life of the animal, followed by the value 2.42 in body weight against body length. Besides these the growth in the upper and lower extermities exceeds that of body length both showing $\alpha$ values larger than 1 .

In head and face, however, the growth was slightly inferior to that of body length showing $\alpha$ value smaller than I.

Of the relative growth of the internal organs against the body weight, the $\alpha$ values of lung and heart are smaller than 1 showing slightly lesser growth than the body weight, while those of spleen, stomach, kidneys and thymus show superior growth rates than the body weight.

The author wishes to express his sincere gratitude to Prof. M. Mori, Dept. of Anatomy, Kyushu University, School of Medicine, Prof. H. Saegusa, Dept. of Anatomy, Kyushu Dental College, for their valuable guidance throughout the entire course of this study as well as for their kindness in revising this manuscript.

\section{References}

1) Donaldson, H. H.: The rat, data and reference table. 1924

2) Huxley, J. S. : Problems of relative growth. 1932

3) Huxley, J. S. \& Teissier, G. : Terminology of relative growth. Nature. Vol. CXXXVII 1936

4) Outhouse, J. Mendel, L. B. : The rate of growth. 1. Its influence on the skeletal development of the albino rat. J. Exp. Zool. Vol. 641933 
5) Shimizu Mitsuo: On the reiative growth of organs of albino rats. The Zoological Magazine Vol. 53 No. 11941

6) Shimizu Mitsuo : [On the relative growth of the body weight in albino rats.] Igaku to Seibutsugaku Vol. 1 No. 31942

7) Shimizu Mitsuo: [A personal opinion on the applicability of the relative growth study in studying general morphology.] Igaku to Seibutsugaku Vol. 5 No. 51944

Fig. 26

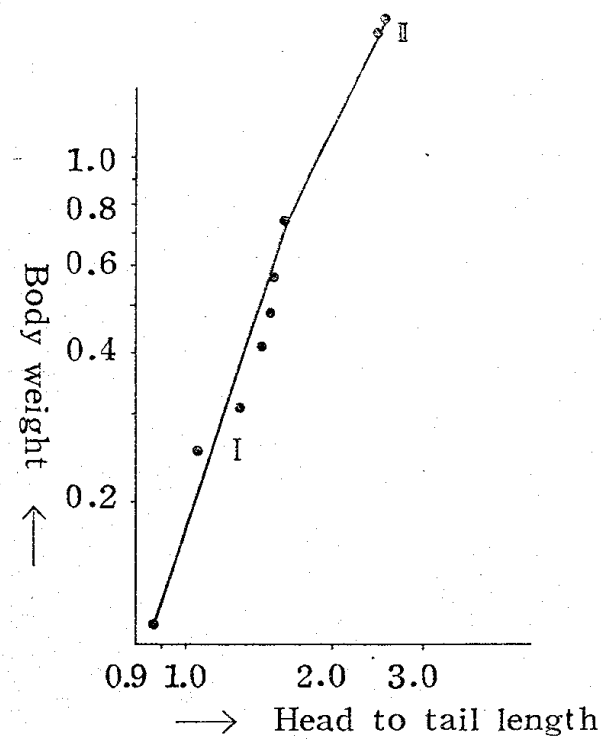

Fig. 27

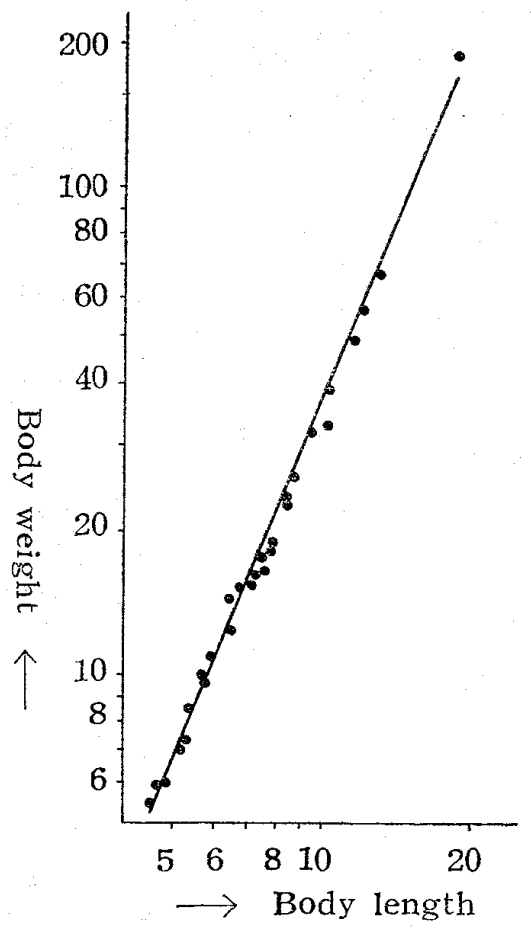

Fig. 28

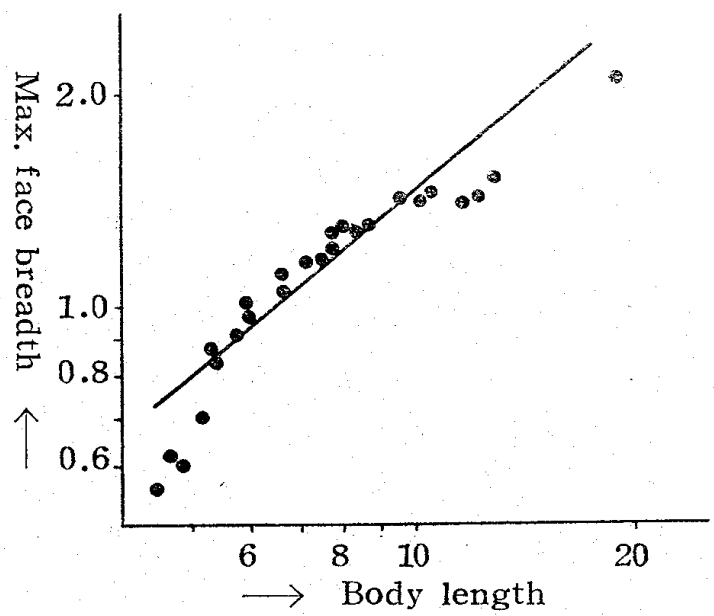

Fig. 29

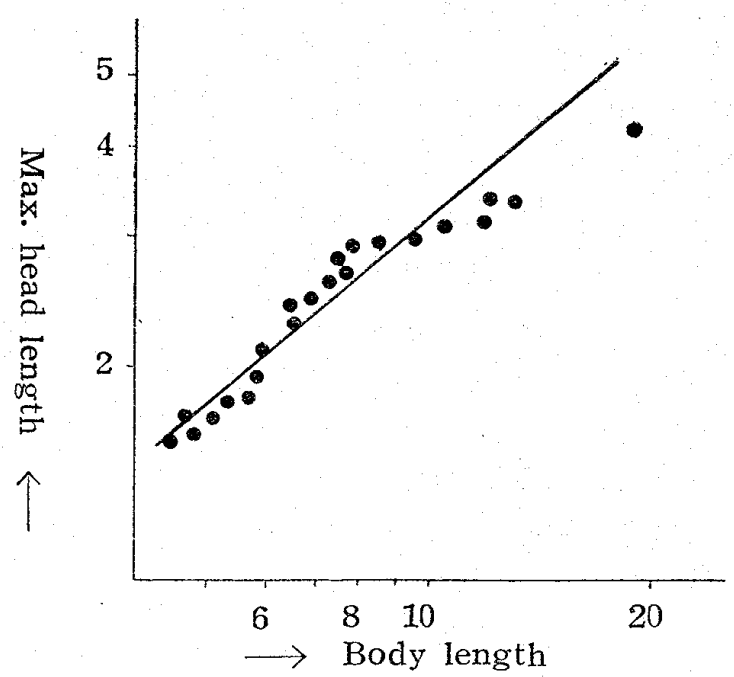

Fig. 30

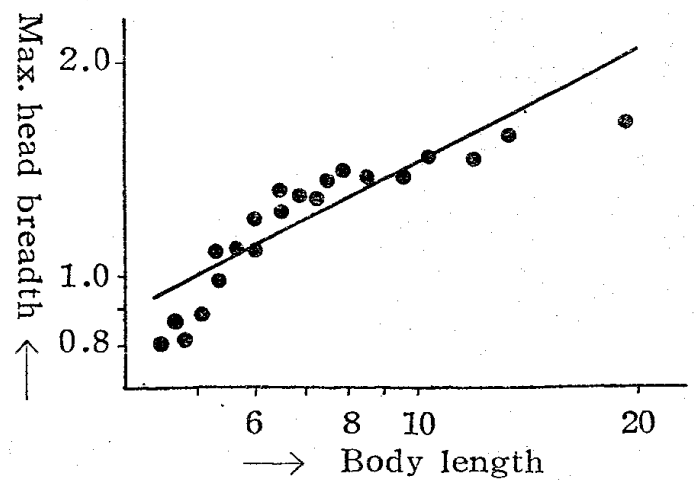


A STUDY ON THE RELATIVE GROWTH IN THE BODY OF ALBINO RATS

Fig. 31 ( a )

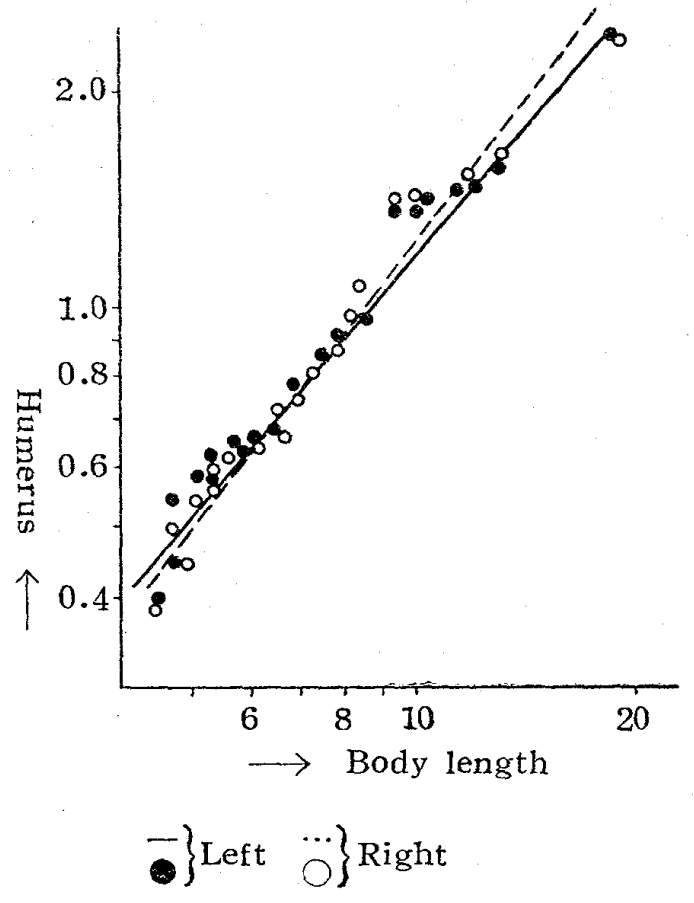

Fig. 31 (b)

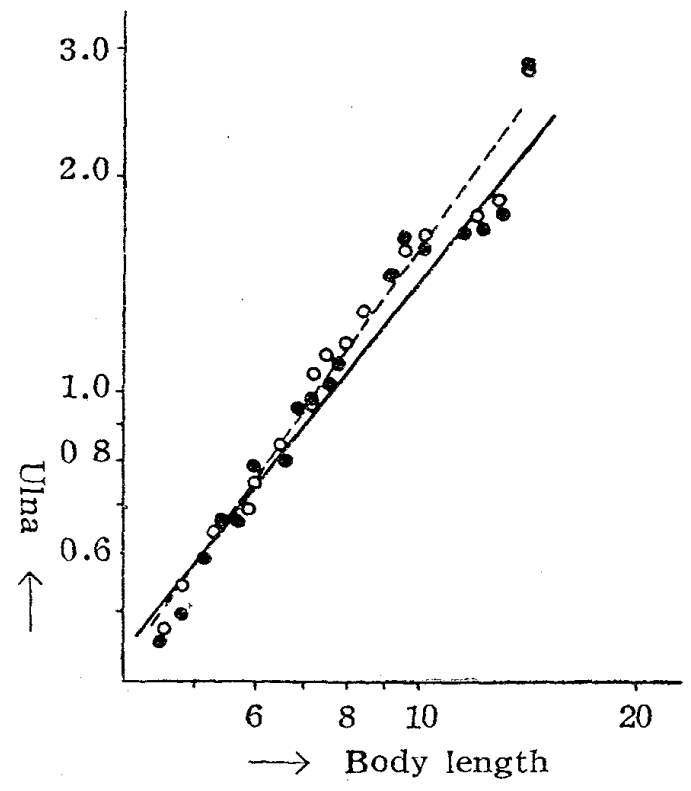

Fig. 31 (c)

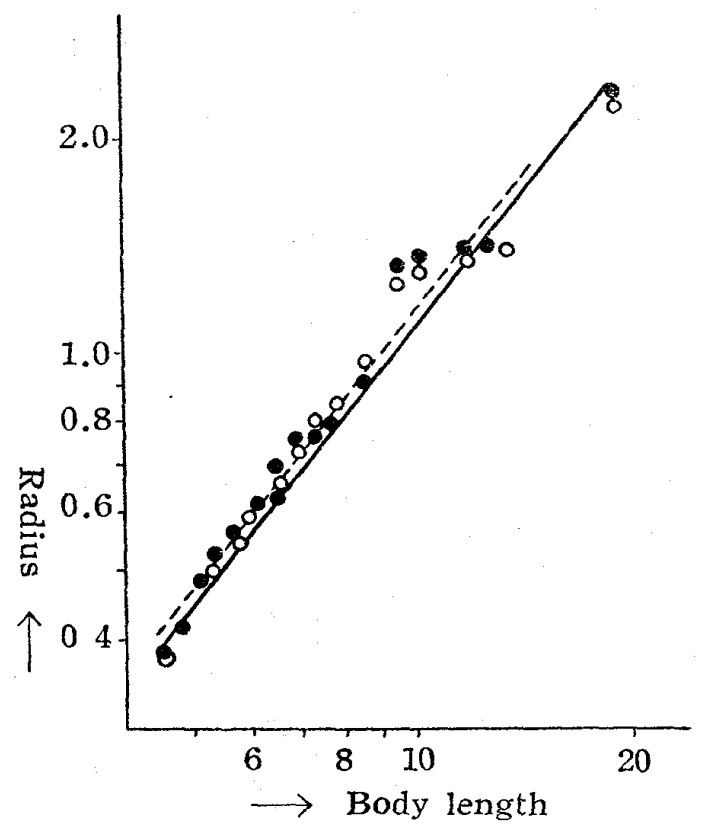

Fig. 32 (a)

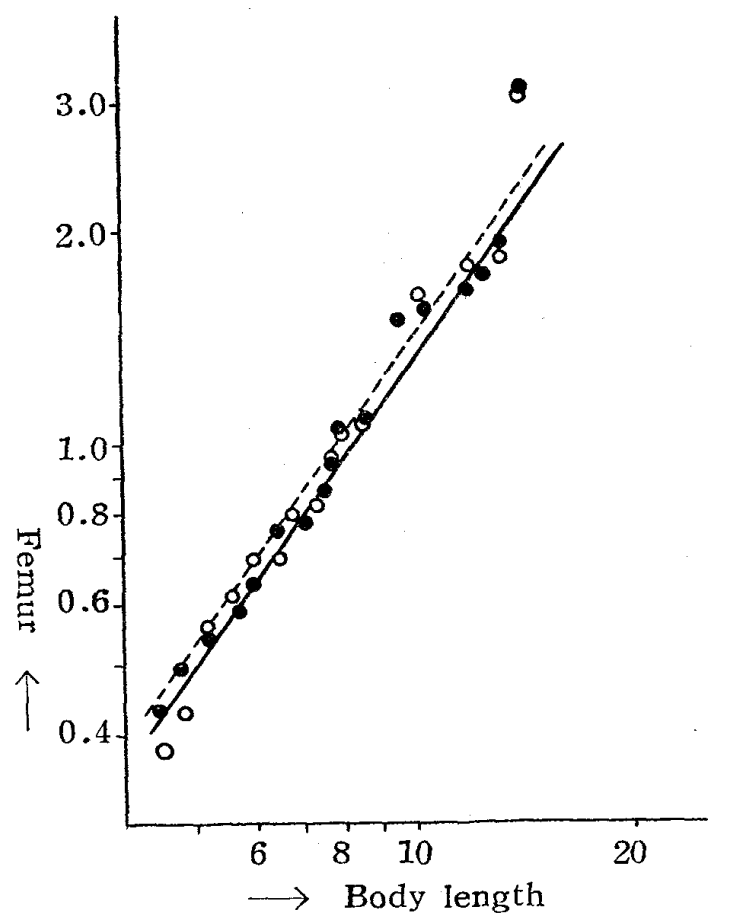


Fig. 32 (b)

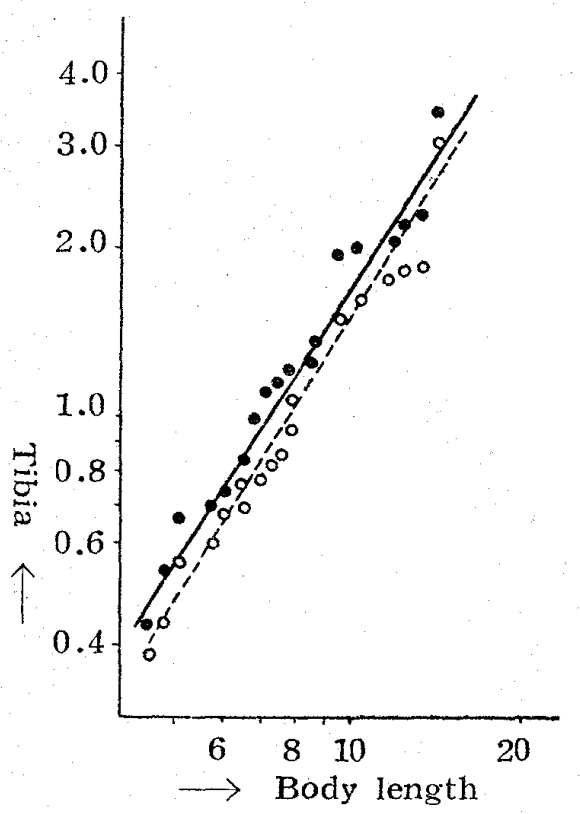

Fig. 34

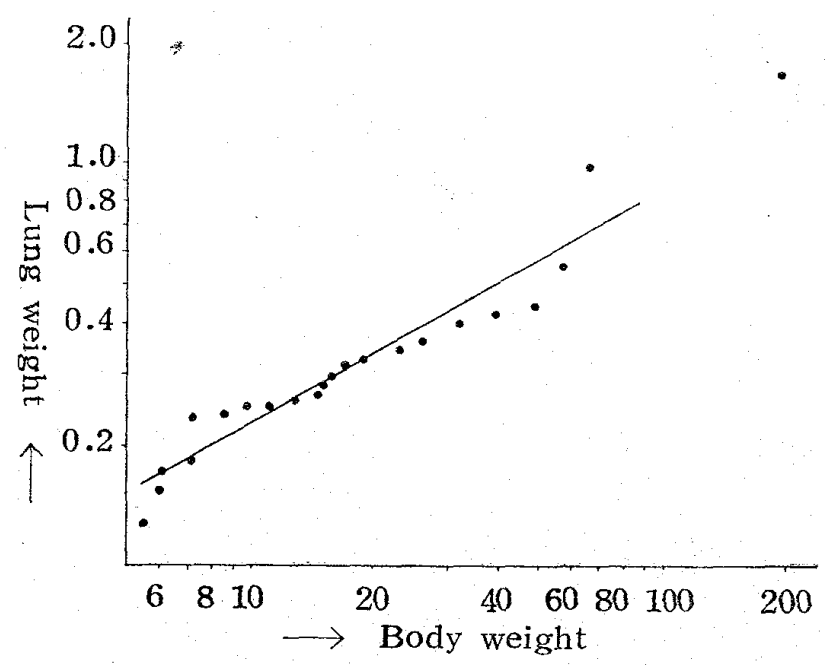

Fig. 35

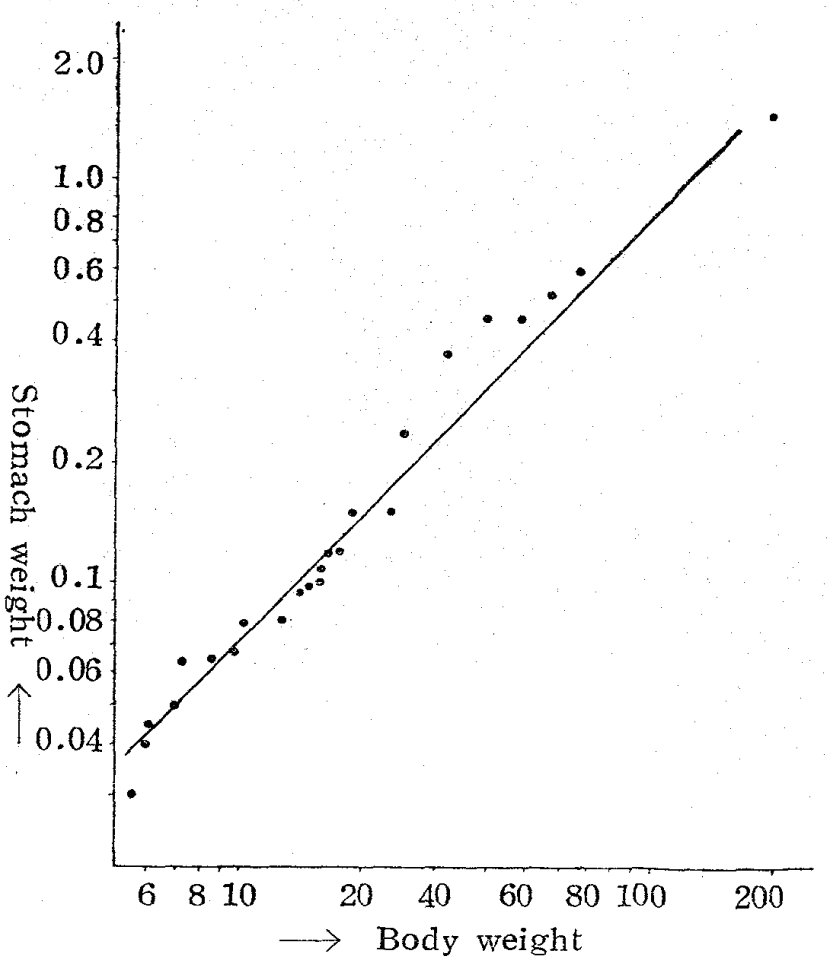

Fig. 33

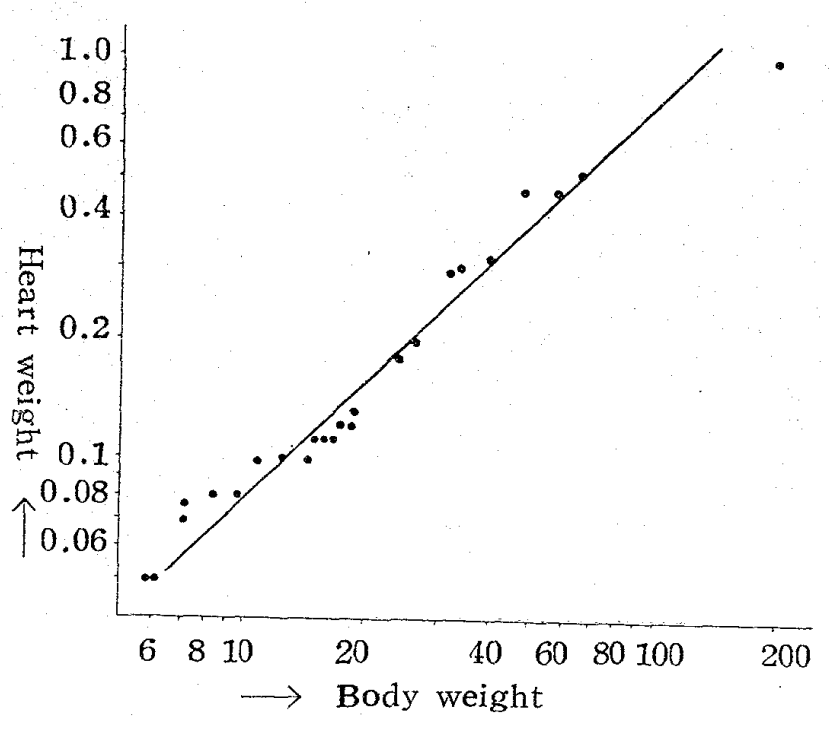


Fig. 36

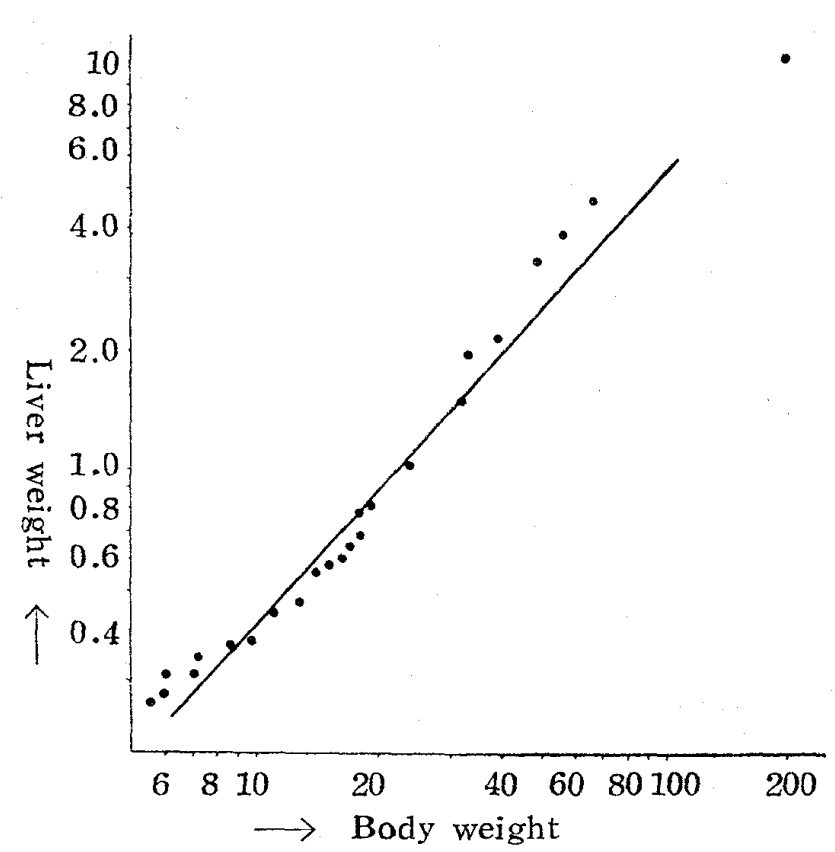

Fig. 37

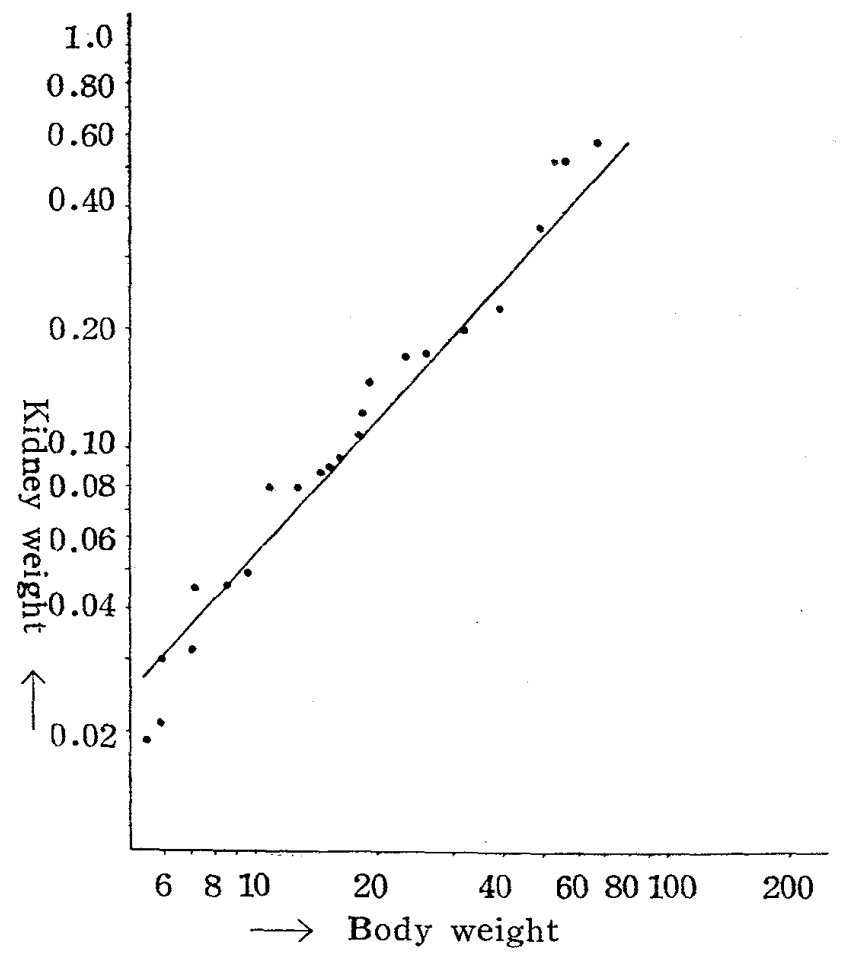

Fig. 38

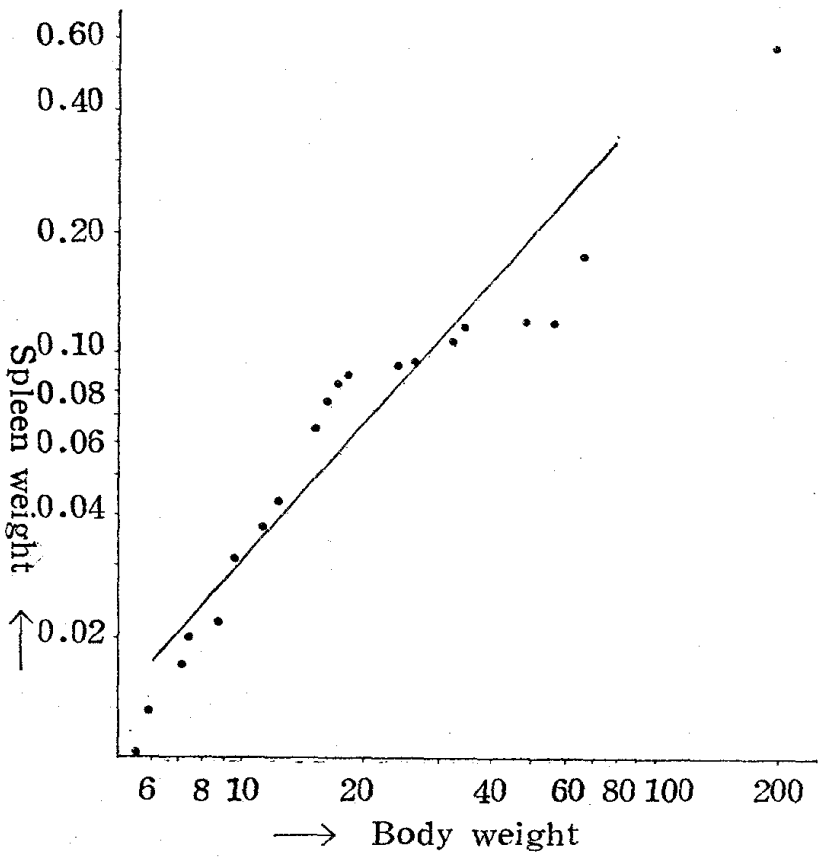

Fig. 39

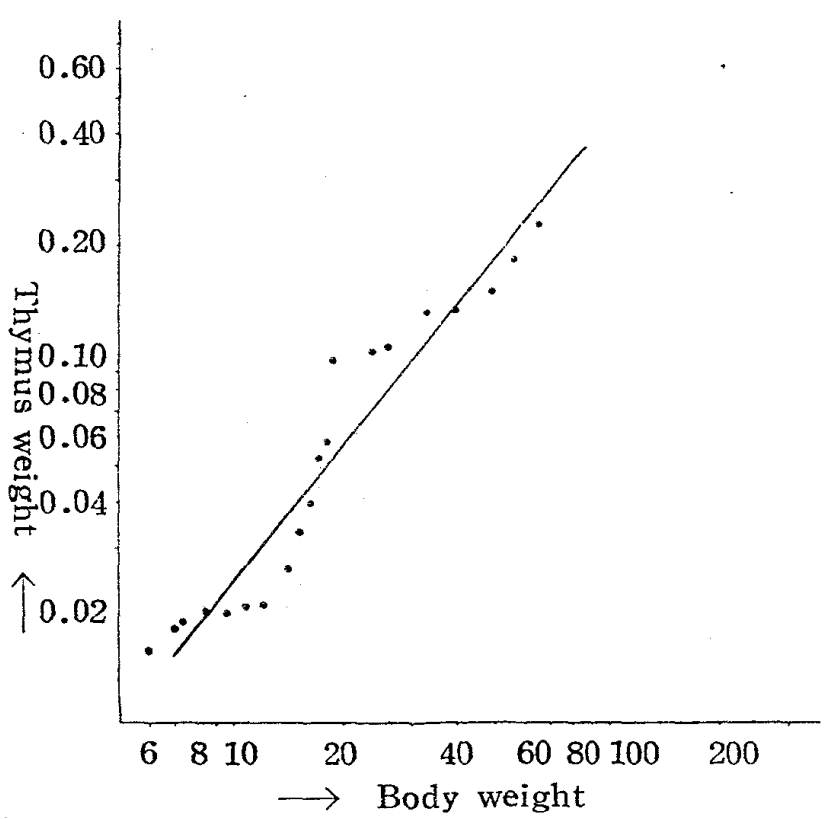


Fig. 40

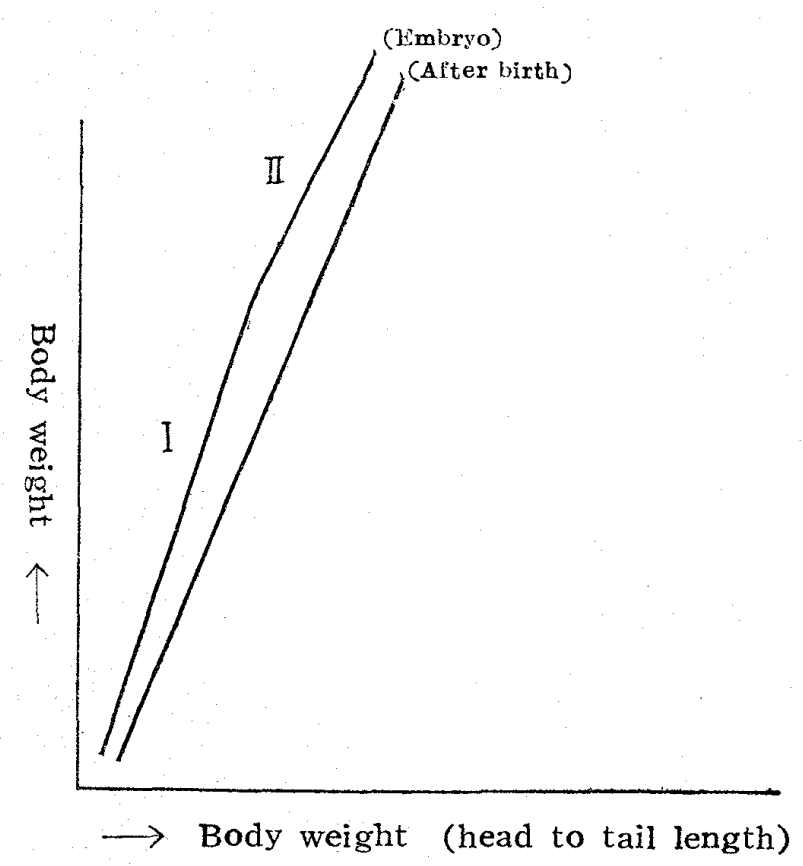

Fig. 42

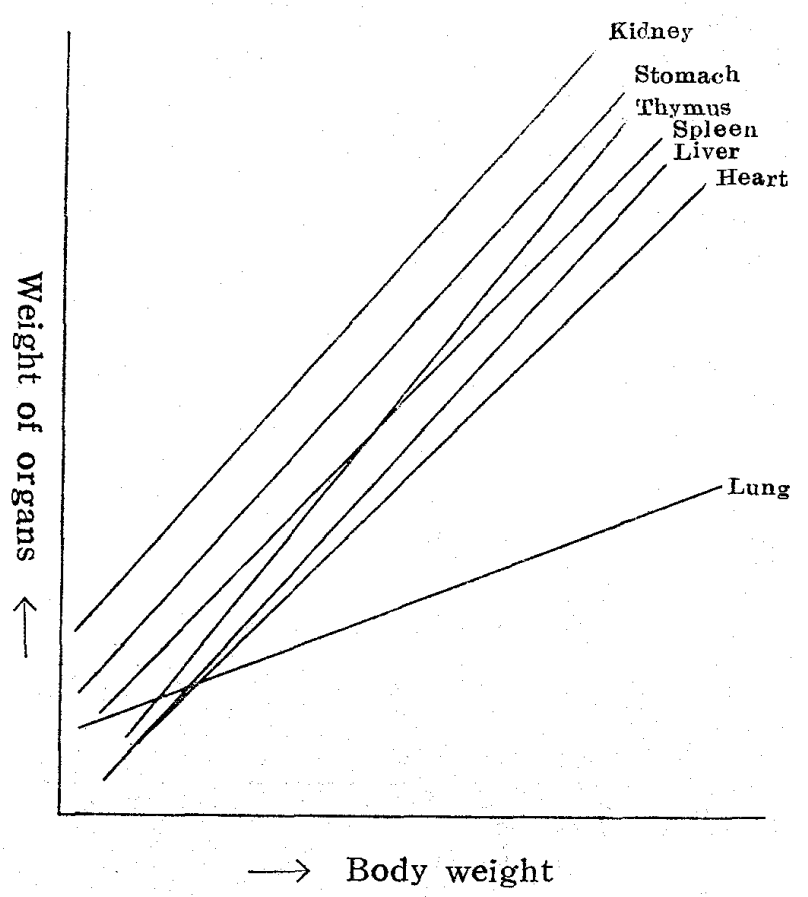

Fig. 41

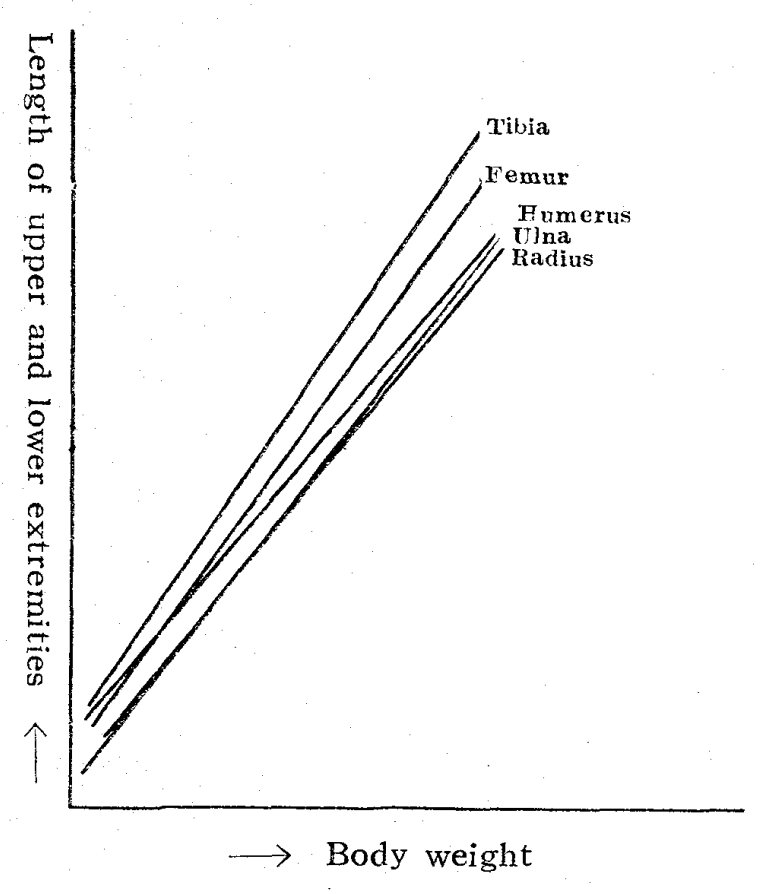

